\title{
B7-H3 suppresses doxorubicin-induced senescence-like growth arrest in colorectal cancer through the AKT/TM4SF1/SIRT1 pathway
}

\author{
Ruoqin Wang ${ }^{1,2,4}$, Linqing Sun², Suhua Xia ${ }^{5}$, Hongya Wu', Yanchao Ma ${ }^{1,2}$, Shenghua Zhan ${ }^{1}$, Guangbo Zhang ${ }^{1,3}$,
} Xueguang Zhang ${ }^{1,3,6}$, Tongguo Shi $\mathbb{B}^{1,3,4}$ and Weichang Chen ${ }^{1,2,3,4,6}$

\begin{abstract}
Emerging evidence suggests that cellular senescence induced by chemotherapy has been recognized as a new weapon for cancer therapy. This study aimed to research novel functions of B7-H3 in cellular senescence induced by a low dose of doxorubicin (DOX) in colorectal cancer (CRC). Here, our results demonstrated that B7-H3 knockdown promoted, while B7-H3 overexpression inhibited, DOX-induced cellular senescence. B7-H3 knockdown dramatically enhanced the growth arrest of CRC cells after low-dose DOX treatment, but B7-H3 overexpression had the opposite effect. By RNA-seq analysis and western blot, we showed that B7-H3 prevented cellular senescence and growth arrest through the AKT/TM4SF1/SIRT1 pathway. Blocking the AKT/TM4SF1/SIRT1 pathway dramatically reversed B7-H3induced resistance to cellular senescence. More importantly, B7-H3 inhibited DOX-induced cellular senescence of CRC cells in vivo. Therefore, targeting B7-H3 or the B7-H3/AKT/TM4SF1/SIRT1 pathway might be a new strategy for promoting cellular senescence-like growth arrest during drug treatment in CRC.
\end{abstract}

\section{Introduction}

Colorectal cancer (CRC) is the third most frequently diagnosed malignancy worldwide ${ }^{1}$. Chemotherapy is a conventional treatment option used to fight against CRC $^{2}$ and has made great contributions to the long-term decline in death rates. Doxorubicin (DOX), a kind of anthracycline drug, is known to be effective against a wide variety of cancers, such as carcinomas, sarcomas, and hematological cancers ${ }^{3}$. Unfortunately, the therapeutic efficacy of DOX is proved to be limited due to its toxicity and resistance mechanisms ${ }^{3,4}$. An increasing number of studies have indicated that low-dose DOX can induce cellular senescence ${ }^{5,6}$, which not only reduces side effects but

\footnotetext{
Correspondence: Tongguo Shi (shitg@suda.edu.cn) or

Weichang Chen (weichangchen@126.com)

${ }^{1}$ Jiangsu Institute of Clinical Immunology, The First Affiliated Hospital of

Soochow University, 708 Renmin Road, Suzhou, China

${ }^{2}$ Department of Gastroenterology, The First Affiliated Hospital of Soochow

University, 188 Shizi Road, Suzhou, China

Full list of author information is available at the end of the article

Edited by S. Tait
}

also prevents tumor growth in vitro and in vivo ${ }^{7,8}$. Hence, an intervention strategy dependent on DOX-induced cellular senescence seems to be a potential option in future CRC treatment.

Cellular senescence, first described by Hayflick and Moorhead, is a physiological phenomenon that involves essentially irreversible growth arrest ${ }^{9}$ and is characterized by morphological alteration, chromatin remodeling, cell cycle arrest, increased senescence-associated $\beta$-galactosidase (SA$\beta$-gal) activity, and a senescence-associated secretory phenotype (SASP) $)^{10,11}$. Cell senescence exists in various precancerous lesions in humans and mice but is reduced in malignant tumors ${ }^{12}$. As a physiological barrier against tumor initiation and progression, cellular senescence is viewed as a natural defense mechanism against tumor progression ${ }^{13,14}$. Therefore, therapy that evokes tumor cellular senescence may be used as a strategy for tumor treatment. A growing body of work has indicated that multiple abnormally expressed genes in cancer cells may participate in the regulation of tumor cellular senescence

\section{(c) The Author(s) 2021}

(c) (i) Open Access This article is licensed under a Creative Commons Attribution 4.0 International License, which permits use, sharing, adaptation, distribution and reproduction cc) in any medium or format, as long as you give appropriate credit to the original author(s) and the source, provide a link to the Creative Commons license, and indicate if changes were made. The images or other third party material in this article are included in the article's Creative Commons license, unless indicated otherwise in a credit line to the material. If material is not included in the article's Creative Commons license and your intended use is not permitted by statutory regulation or exceeds the permitted use, you will need to obtain permission directly from the copyright holder. To view a copy of this license, visit http://creativecommons.org/licenses/by/4.0/. 
induced by drugs ${ }^{8}$. For instance, the chromatin remodeling enzyme ATRX is a critical regulator of therapy-induced senescence $^{15}$. The knockdown of TRIB2, a member of the tribble family, promoted DOX-induced senescence of SW48 and LoVo CRC cells in a p21-dependent manner ${ }^{16}$. Zhu and coworkers showed that nuclear receptor HNF4 $\alpha$ overexpression enhanced cellular senescence caused by DOX in prostate cancer cells ${ }^{17}$. Nevertheless, the underlying molecular mechanism of the regulation of DOX-induced senescence has not yet been well elucidated.

B7-H3 (B7 homolog 3), also known as CD276, serves as a member of the B7-CD28 superfamily ${ }^{18}$ and is overexpressed in a variety of malignancies, such as CRC, pancreatic cancer, prostate cancer, and ovarian cancer ${ }^{19-22}$. Due to conflicting costimulatory and coinhibitory functions, the immunologic function of B7-H3 remains controversial $^{23}$. Aside from its immunologic function, B7-H3 has been reported to be involved in tumorigenesisassociated nonimmunological functions such as proliferation, metastasis, metabolism, and angiogenesis ${ }^{24-27}$. Moreover, $\mathrm{B} 7-\mathrm{H} 3$ exerts a great impact on the regulation of chemoresistance in several malignancies, including $\mathrm{CRC}^{28,29}$. Although senescence-associated exosomes of human prostate cancer cells are enriched in the B7-H3 protein $^{30}$, few studies have explored the detailed roles and molecular mechanisms of $\mathrm{B} 7-\mathrm{H} 3$ in modulating cellular senescence in cancers such as CRC. Hence, the aim of the current study was to explore whether B7-H3 regulated cellular senescence and growth arrest in CRC cells treated with a low dose of DOX in an attempt to optimize chemotherapy-induced senescence therapies associated with $\mathrm{B} 7-\mathrm{H} 3$.

\section{Materials and methods CRC clinical samples and immunohistochemistry (IHC) assay}

A total of 54 pairs of CRC primary tumor tissues and adjacent normal tissues were obtained from CRC patients who received surgical resection at the First Affiliated Hospital of Soochow University (Suzhou, China). This study was approved by the Institutional Review Board of Soochow University, and informed consent was obtained from the patients. Detailed clinicopathological information is provided in Supplementary Table 1. An IHC assay and the scoring criteria were conducted as previously described $^{26}$. Briefly, after deparaffinization and rehydration, tissues were treated with antigen retrieval using $10 \mathrm{mM}$ sodium citrate buffer ( $\mathrm{pH}$ 6.0), followed by incubation with B7-H3 antibody (R\&D Systems, MN, USA, \#AF1027, 1:100) and TM4SF1 antibody (Abcam, Cambridge, UK, \#ab113504, 1:500) overnight at $4{ }^{\circ} \mathrm{C}$. We used the semiquantitative immunoreactive score (IRS) system to analyze the B7-H3 and TM4SF1 immunostaining. The intensity of immunostaining was scored using the following criteria: 0 , negative; 1 , weak; 2, moderate; 3, strong. Besides, the percentage of immunoreactive cells was scored using the following criteria:1, (0-25\%); 2, (26-50\%); 3, (51-75\%); and 4, (76-100\%). Final scores were calculated by multiplying the scores of two parts in the same section; the scores ranged from 0 to 12 . Two pathologists (Dr. Xia and Dr. Zhan) reviewed blindly and scored IHC staining independently for each sample.

\section{Cell culture, transfection, and infection}

The cell lines HCT116 and RKO were obtained from the American Type Culture Collection (ATCC, Manassas, VA, USA). These cells were cultured in DMEM (Biological Industries, Beit Haemek, Israel) plus 10\% fetal bovine serum (FBS, Biological Industries) and $1 \%$ penicillinstreptomycin (Beyotime, Shanghai, China, \#C0222) at 5\% $\mathrm{CO}_{2}$ and $37^{\circ} \mathrm{C}$.

Cells were transfected with TM4SF1 siRNA, SIRT1 siRNA or control siRNA (RiboBio, Guangzhou, China) using Lipofectamine 2000 (Invitrogen, Carlsbad, CA, USA) according to the manufacturer's protocol. The TM4SF1 plasmid was obtained from GeneCopoeia (Guangzhou, China, \#EX-Z2186-Lv105). TM4SF1overexpressing HCT116 or RKO cells were generated according to the manufacturer's protocol. Stable B7-H3overexpressing HCT116 or RKO cells (B7-H3 HCT116 or B7-H3 RKO) and knockdown HCT116 or RKO cells (shB7-H3 HCT116 or shB7-H3 RKO) were described earlier $^{27}$.

\section{Induction of cell senescence}

For induction of cell senescence by DOX (Aladdin, Shanghai, China, \#25316-40-9), HCT116 and RKO cells were treated with a variety of concentrations of DOX (HCT116: $100 \mathrm{nM}$, RKO: $50 \mathrm{nM}$ ) and cultured in DMEM with $5 \%$ FBS for $96 \mathrm{~h}$. The established cellular senescence models were used for further analysis.

\section{Western blot analysis}

Cells were lysed in RIPA lysis buffer (Beyotime, \#P0013D) at $4{ }^{\circ} \mathrm{C}$. Protein concentration was examined using the BCA method (Beyotime, \#P0010). Total protein $(30 \mu \mathrm{g})$ was separated via $10 \%$ SDS-PAGE (Beyotime, \#P0012AC) and transferred onto 0.45- $\mu \mathrm{m}$ PVDF membranes (GE Healthcare Life Science, Germany). After blocking with 5\% BSA (Fcmacs, Nanjing, China, \#FMSWB021) for $1 \mathrm{~h}$, the membranes were incubated at $4{ }^{\circ} \mathrm{C}$ overnight with one of the antibodies listed in Supplementary Table 2 . The next day, membranes were washed and incubated with the corresponding HRP-conjugated secondary antibodies (Beyotime) for $1 \mathrm{~h}$ at room temperature. Finally, immunoreactive bands were detected with ECL reagents (NCM Biotech, Suzhou, China, 
\#10100) using a ChemiDocTM MP Imaging System (BioRad).

RNA extraction and real-time quantitative PCR (RT-qPCR)

Total RNA was isolated from CRC cells using TRIzol (TransGen Biotech, Beijing, China, \#ER501-01) in accordance with the manufacturer's instructions. Then, cDNA was synthesized using PrimeScript RT Master Mix (Takara, Shiga, Japan, \#RR036A), and RT-qPCR reactions were performed using SYBR Green Master Mix (Vazyme, Nanjing, China, \#Q121-02-AA) according to the manufacturer's instructions. The reaction conditions were described as previously ${ }^{27}$. $\beta$-actin was used as a constitutive control. PCRs for each sample were conducted in triplicate. The primers were designed based on GenBank sequences and are listed in Supplementary Table 3.

\section{SA- $\beta$-gal staining}

Cells were subjected to SA- $\beta$-gal staining using the Senescent $\beta$-galactosidase Staining Kit (Beyotime, \#C0602) according to the manufacturer's protocol. Briefly, cells were washed with PBS and fixed with the fixative solution for $15 \mathrm{~min}$. Then, the cells were incubated with the staining solution overnight at $37{ }^{\circ} \mathrm{C}$ without $\mathrm{CO}_{2}$. Finally, green-stained positive cells were photographed and counted from three different locations per well by an inverted microscope.

\section{SAHF detection}

Senescence-associated heterochromatin foci (SAHF), visualized as DAPI-dense foci, is a feature of some senescent cells ${ }^{10}$. To identify the SAHFs, cells were fixed with $4 \%$ paraformaldehyde for $15 \mathrm{~min}$ and permeabilized with $1 \%$ Triton X-100 for $10 \mathrm{~min}$. Then, the cells were stained with DAPI (Invitrogen, USA, \#S36939) for $5 \mathrm{~min}$. DAPI-stained nuclei with blue fluorescence were finally photographed and counted from three different locations per well under a confocal laser scanning microscope (Olympus FLUOVIEW FV1000).

\section{CCK-8 assay}

Cell viability was determined by using the Cell Counting Kit-8 (Dojindo, Kumamoto, Japan, \#CK04) assay. Briefly, CRC cells were seeded in 96-well plates $\left(5 \times 10^{3}\right.$ cells/ well). After the cells were treated with DOX for 24, 48, 72 or $96 \mathrm{~h}, 10 \mu \mathrm{l} \mathrm{CCK-8} \mathrm{solution} \mathrm{was} \mathrm{added} \mathrm{into} \mathrm{each} \mathrm{well} \mathrm{for}$ $4 \mathrm{~h}$ at $37^{\circ} \mathrm{C}$. The absorbance in each well was measured at a wavelength of $450 \mathrm{~nm}$.

\section{Colony formation assay}

For the colony formation assay, cells were seeded into 6well plates $\left(2 \times 10^{3}\right.$ cells/well $)$ and incubated for 14 days. The colonies were fixed in $4 \%$ paraformaldehyde for $15 \mathrm{~min}$, washed with PBS, and stained with crystal violet
(Beyotime, \#C0121) for $15 \mathrm{~min}$. Finally, the number of colonies was photographed and counted from three different wells.

\section{Cell cycle analysis}

Cells were collected, washed with cold PBS twice and fixed with $70 \%$ ethanol containing $0.5 \%$ FBS overnight at $-20{ }^{\circ} \mathrm{C}$. Cells were then treated with cell cycle assays (Fcmacs, Nanjing, China) according to the manufacturer's protocol. Cell cycle distribution was measured by the Becton-Dickinson FACScan System (Franklin Lakes, NJ, USA). A total of 10,000 events were collected per sample.

\section{RNA-seq assay}

For RNA-Seq analysis, total RNA was extracted from senescent shB7-H3 RKO cells or control cells as described above. The quantity and quality of the total RNA were determined by measuring the OD 260/280 ratio, and samples with ratios between 1.8 and 2.1 were confirmed as having good integrity. The RNA-seq assay was carried out by GENEWIZ Biotech Co. (Suzhou, China).

\section{Xenograft tumor}

The animal assay was approved by the Institutional Animal Care and Use Committee of Soochow University (Suzhou, China). Six to eight-week-old female BALB/C nude mice were purchased from the Shanghai Laboratory Animal Center (Shanghai, China). All mice were randomly assigned to different groups. To create the xenograft tumor model, $5 \times 10^{6}$ B7-H3 HCT116 cells, EV HCT116 cells, shB7-H3 HCT116 cells or sh-NC HCT116 cells were injected subcutaneously into the right flank of the mice, respectively. To investigate the effect of B7-H3 on DOXinduced cellular senescence in vivo, mice with xenograft tumors were intraperitoneally injected with DOX at a low dose of $4 \mathrm{mg} / \mathrm{kg}$ every other day. The volume of xenograft tumors was measured every 3 days by using digital calipers. After 2 weeks, all mice were sacrificed, and tumor samples were weighed. In addition, the xenograft tumor tissues were studied by SA- $\beta$-gal staining and IHC assay. All results of animal experiments were obtained blindly.

\section{Statistical analysis}

All calculations were analyzed using GraphPad Prism 5.0 (La Jolla, CA, USA). The significance of differences between two groups was determined by Student's $t$-test. All data are expressed as at least three biological replicates. A value of $P<0.05$ was considered statistically significant.

\section{Results}

B7-H3 suppresses cellular senescence and senescence-like cell growth arrest in CRC cells

To identify the functional role of B7-H3 in low-dose DOX-induced senescence in CRC cells, we used stable 
B7-H3 knockdown (shB7-H3) HCT116 or RKO cells that we described previously ${ }^{27}$. B7-H3 knockdown stably inhibited $\mathrm{B} 7-\mathrm{H} 3$ mRNA and protein expression in HCT116 or RKO cells (Supplementary Fig. S1A and Fig. 1A). We treated shB7-H3 HCT116 or RKO cells with a low dose of DOX (100 nM for HCT116 cell, and $50 \mathrm{nM}$ for RKO cell) for $96 \mathrm{~h}$ and found that the protein expression of p21 was markedly increased under B7-H3 knockdown conditions (Fig. 1B). B7-H3 knockdown had no effect on the protein expression of p53 in CRC cells treated with low-dose DOX (Fig. 1B). The percentage of SA- $\beta$-gal- and SAHF-positive cells was obviously increased in shB7-H3 CRC cells treated with low-dose DOX (Fig. 1C-E). Moreover, we observed that B7-H3 knockdown dramatically enhanced the growth arrest of CRC cells treated with low-dose DOX, as detected by both CCK- 8 and colony formation assays (Fig. 1F, G). Additionally, the flow cytometry-based cell cycle assay results showed that B7-H3 knockdown further aggravated G2/M phase arrest in HCT116 and RKO cells treated with lowdose DOX (Fig. 1H).

In complementary loss-of-function studies, HCT116 or RKO cells stably overexpressing B7-H3 were used (Supplementary Fig. S1B and Fig. 2A). B7-H3 overexpression significantly decreased the protein expression of p21 in HCT116 and RKO cells treated with low-dose DOX but had no effect on p53 protein expression (Fig. 2B). Moreover, $\mathrm{B} 7-\mathrm{H} 3$ overexpression obviously reduced the percentage of SA- $\beta$-gal- and SAHF-positive CRC cells treated after low-dose DOX treatment (Fig. 2C-E). Furthermore, the effects of $\mathrm{B} 7-\mathrm{H} 3$ overexpression on senescence-like growth arrest and cell cycle arrest were evaluated, and the results revealed that after the elevation of $\mathrm{B} 7-\mathrm{H} 3$, low-dose DOX-induced CRC cell growth arrest and G2/M cell cycle arrest were alleviated (Fig. 2F-H). Based on the obtained results, we conclude that $\mathrm{B} 7-\mathrm{H} 3$ is involved in the resistance to low-dose DOX-induced cell senescence in CRC.

\section{B7-H3 promotes the expression of TM4SF1 in senescent CRC cells}

To determine the reasons why B7-H3 inhibited cellular senescence and senescence-like growth arrest in CRC, we performed RNA-seq analysis to identify the differentially expressed genes (DEGs) in shB7-H3 RKO cells treated with low-dose DOX. First, DEG analysis yielded a list of 439 genes that were significantly dysregulated (139 downregulated and 300 upregulated, $P<0.05$, Fig. 3A). Among these, 22 DEGs with logarithm of fold change $(|\log \mathrm{FC}|)>10$ were identified (Fig. 3B, C and Supplementary Table 4). Then, we noticed transmembrane-4 Lsix family member-1 (TM4SF1) in the top differentially expressed genes. TM4SF1, a signal transducer, has been reported to regulate cell proliferation, migration, invasion, and chemoresistance in multiple cancers ${ }^{31-33}$. Therefore, we hypothesized that $\mathrm{B} 7-\mathrm{H} 3$ might affect the cellular senescence of CRC cells by modulating TM4SF1. The results of RT-qPCR and western blot assays showed that the mRNA and protein expression of TM4SF1 were significantly downregulated in shB7-H3 CRC cells but upregulated in B7-H3-overexpressing cells (Fig. 3D-G). To conclude, TM4SF1 does factor into B7-H3-associated resistance to low-dose DOX-induced senescence.

\section{TM4SF1 is highly expressed and positively correlates with B7-H3 expression in CRC clinical tissues}

Next, we investigated the correlation between the levels of B7-H3 and TM4SF1 in CRC clinical samples by IHC staining. Clearly, TM4SF1, the same as B7-H3, was strongly upregulated in CRC tissue samples compared to that in normal tissues (Fig. 3H, I and Supplementary Fig. S2A). Additionally, the expression of TM4SF1 was increased in advanced (III and IV stages) CRC patients compared to early stage (I and II) patients (Fig. 3J), sharing the same tendency with the expression of B7-H3 (Supplementary Fig. S2B). Moreover, we selected 27 cases (50\%) with low expression of B7-H3 (<median value) and 27 cases $(50 \%)$ with high expression (>median value). The high B7-H3 expression group showed higher TM4SF1 expression than the low group (Fig. 3K). More importantly, the expression level of TM4SF1 showed an obvious positive association with B7-H3 levels in CRC tissue samples (Fig. 3L). In addition, samples of CRC with lymph node metastasis showed higher B7-H3 and TM4SF1 expression than those without lymph node metastasis (Supplementary Fig. S2C, D).

\section{B7-H3 regulates cellular senescence via TM4SF1}

The above-described results suggested that TM4SF1 may be a key downstream target molecule of $\mathrm{B} 7-\mathrm{H} 3$ in CRC cells treated with low-dose DOX. Hence, we further investigated whether the effect of $\mathrm{B} 7-\mathrm{H} 3$ on cellular senescence was TM4SF1 dependent. The Western blot results showed that TM4SF1 overexpression upregulated p21 protein levels in low-dose DOX-induced senescent shB7-H3 HCT116 and RKO cells (Fig. 4A). In addition, as shown in Fig. 4B-E, TM4SF1 overexpression partially reversed the effect of $\mathrm{B} 7-\mathrm{H} 3$ knockdown on increased percentages of SA- $\beta$-gal- and SAHF-positive cells in CRC cells treated with low-dose DOX (Fig. 4B-E). Moreover, the increase in cell growth arrest and G2/M phase arrest were partially alleviated by TM4SF1 overexpression in shB7-H3 CRC cells treated with low-dose DOX (Fig. $4 \mathrm{~F}-\mathrm{H})$. Moreover, we filtered and obtained the most effective TM4SF1 siRNA-2 among three commercial siRNAs targeting TM4SF1, as detected by both RTqPCR and western blot assays (Supplementary Fig. S3A, B). Treatment with TM4SF1 siRNA-2 led to a significant increase in the percentages of SA- $\beta$-gal- and 
A

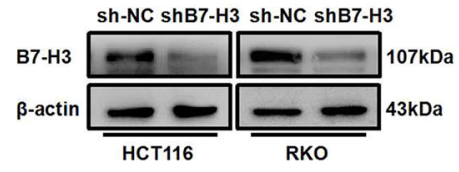

C

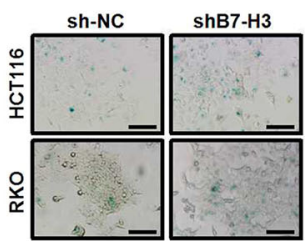

D

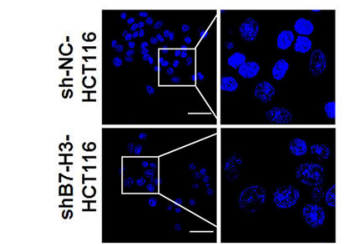

$\mathbf{F}$

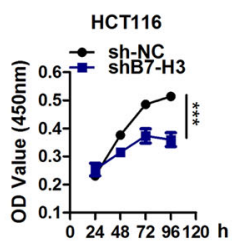

\section{B}
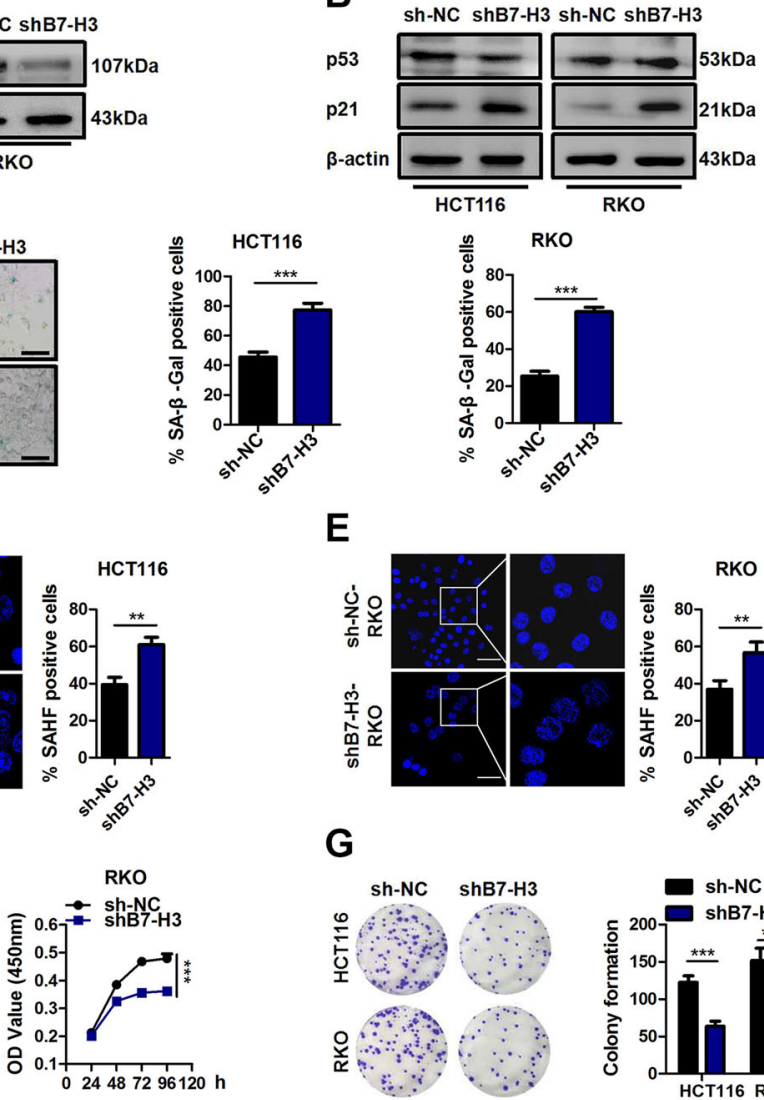

E
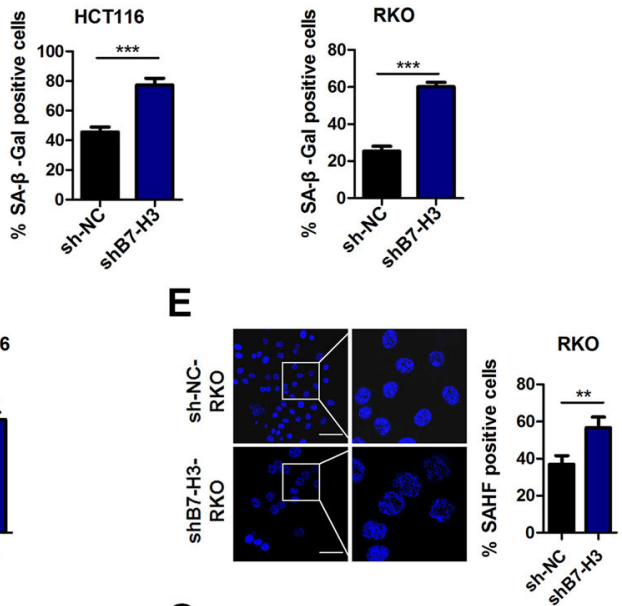

G

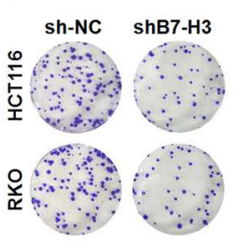

H
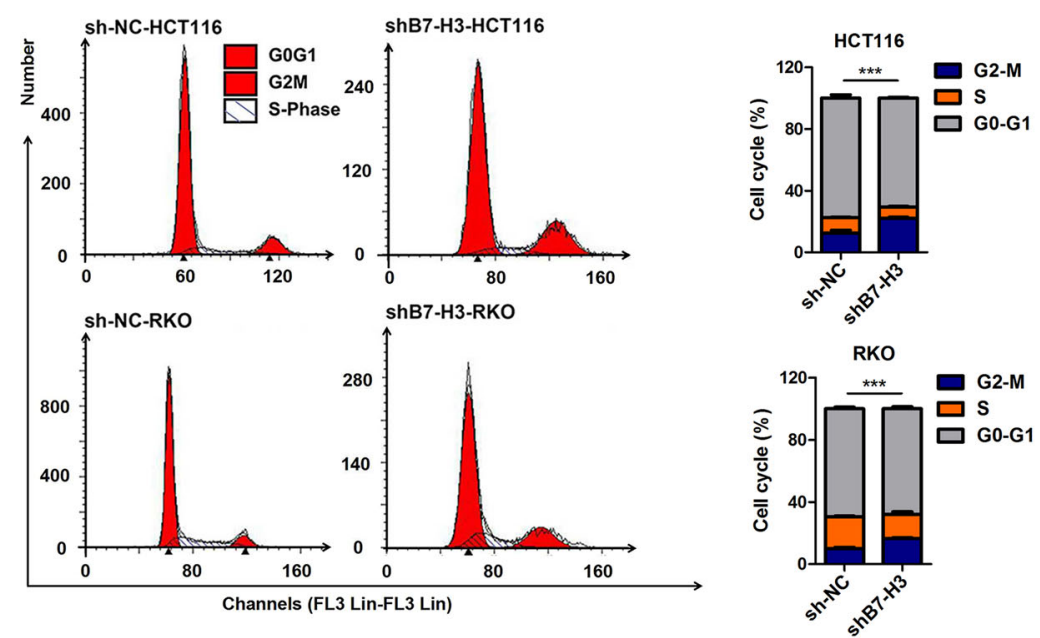

Fig. 1 Knockdown of B7-H3 promotes DOX-induced senescence. A Western blot analysis of B7-H3 in CRC stable cell lines with B7-H3 inhibition (shB7-H3) or their control cell lines (sh-NC). $\beta$-Actin served as a loading control. B Western blot analysis of p21 and p53 in sh-NC cells or shB7-H3 CRC cells treated with DOX. $\beta$-Actin served as a loading control. C SA- $\beta$-Gal activity of sh-NC cells or shB7-H3 CRC cells treated with DOX was examined. Scale bar, $100 \mu \mathrm{m}$. One representative image from three reproducible experiments is shown. The percentages of SA- $\beta$-gal-positive cells are shown in the bar graph. D, E SAHF activity of sh-NC cells or shB7-H3 HCT116 cells and RKO cells with DOX treatment was examined. Scale bar, $50 \mu m$. One representative image from three reproducible experiments is shown. The percentages of SAHF-positive cells are shown in the bar graph. $\mathbf{f}$ Cell viability in sh-NC cells or shB7-H3 CRC cells treated with DOX after 24, 36, 48, and $96 \mathrm{~h}$ was examined by CCK-8 assays. G The colony formation of shNC cells or shB7-H3 CRC cells treated with DOX was examined. One representative image from three reproducible experiments is shown. The number of colonies is shown in the bar graph. $\mathbf{H}$ Cell cycle analysis by PI staining in sh-NC cells or shB7-H3 CRC cells with DOX treatment was examined through flow cytometry. The data represent the means \pm SEM. ${ }^{*} P<0.05 ;{ }^{* * *} P<0.001$. 
A

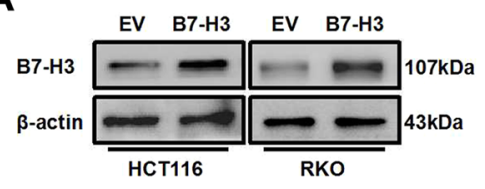

B

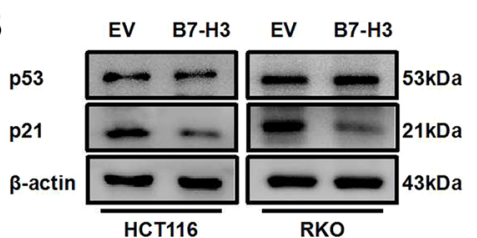

C

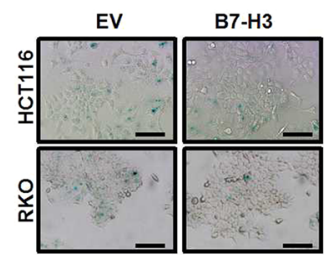

D
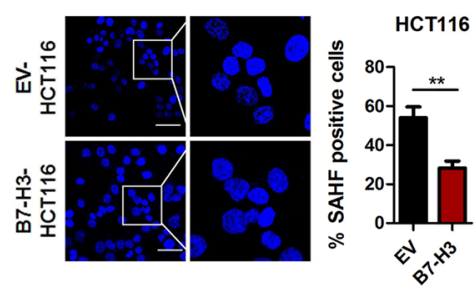

F

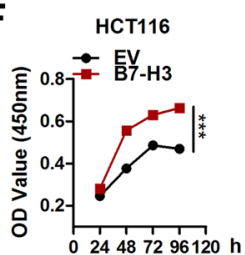

RKO

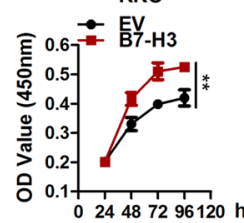

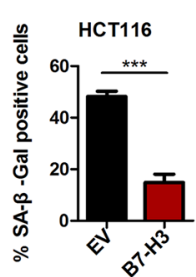

E

\section{E}
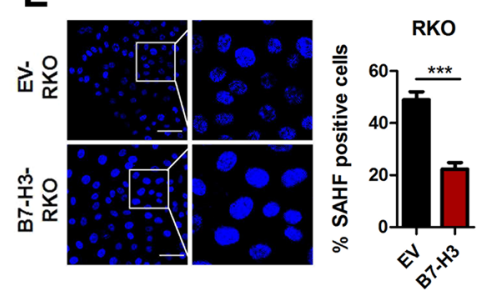

G

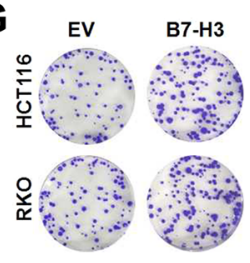

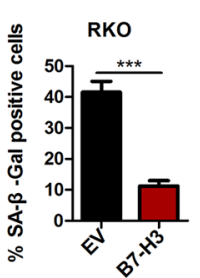

H

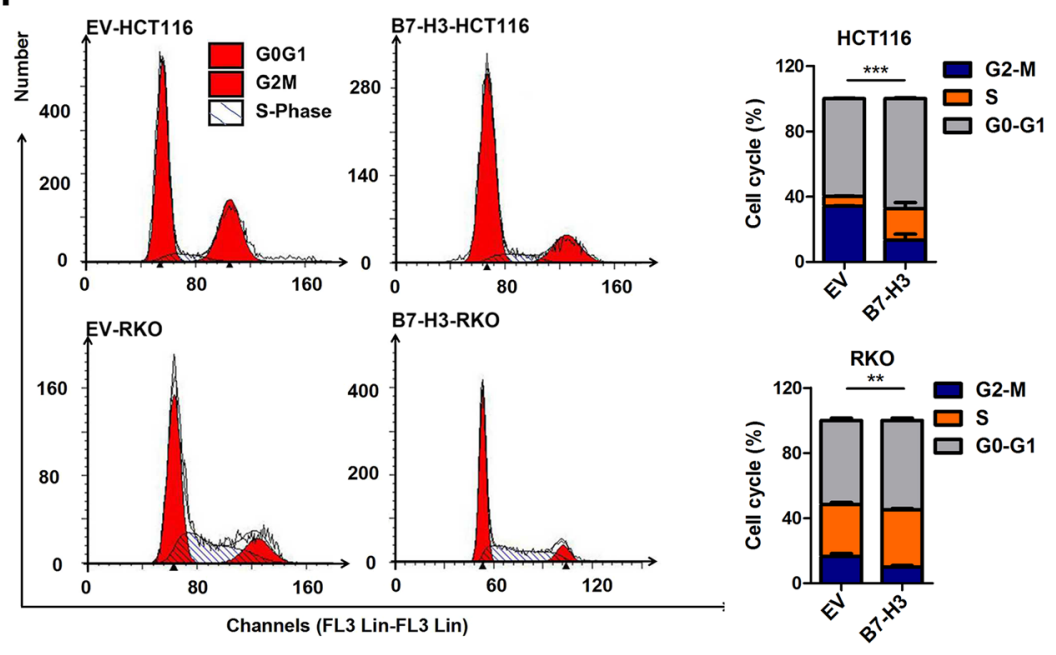

Fig. 2 Overexpression of B7-H3 attenuates the DOX-induced senescence. A Western blot analysis of B7-H3 in CRC stable cell lines with B7-H3 overexpression (B7-H3) or their control cell lines (EV). $\beta$-Actin served as a loading control. B Western blot analysis of p21 and p53 in EV cells or B7-H3 CRC cells treated with DOX. $\beta$-Actin served as a loading control. C SA- $\beta$-Gal activity of EV cells or B7-H3 CRC cells treated with DOX was examined. Scale bar, $100 \mu \mathrm{m}$. One representative image from three reproducible experiments is shown. The percentages of SA- $\beta$-gal-positive cells are shown in the bar graph. D, E SAHF activity of EV cells or B7-H3 HCT116 cells and RKO cells with DOX treatment was examined. Scale bar, $50 \mu \mathrm{m}$. One representative image from three reproducible experiments is shown. The percentages of SAHF-positive cells are shown in the bar graph. $\mathbf{F}$ Cell viability in EV cells or B7-H3 CRC cells treated with DOX after 24, 36, 48, and $96 \mathrm{~h}$ was examined by CCK-8 assays. G The colony formation of EV cells or B7-H3 CRC cells treated with DOX was examined. One representative image from three reproducible experiments is shown. The number of colonies is shown in the bar graph. $\mathbf{H}$ Cell cycle analysis by PI staining in EV cells or B7-H3 CRC cells with DOX treatment was examined through flow cytometry. The data represent the means \pm SEM. ${ }^{*} P<0.05 ;{ }^{* * *} P<0.001$. 


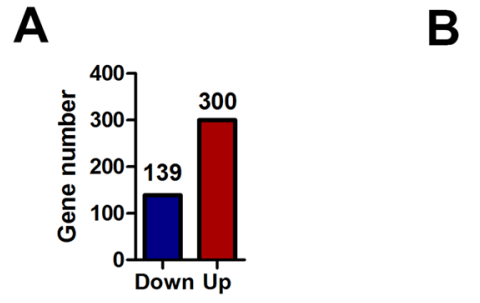

D

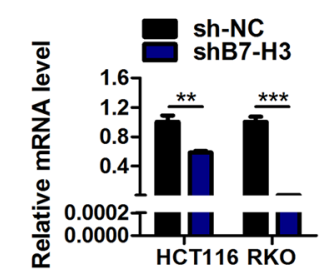

$\mathbf{F}$

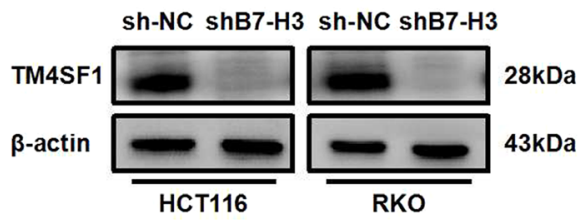

H

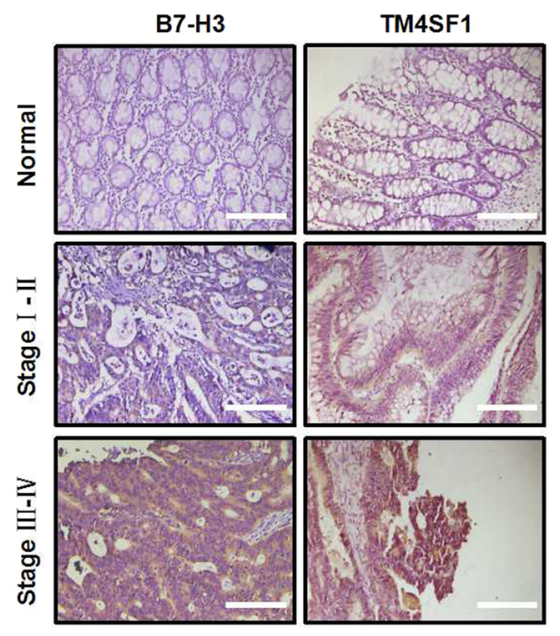

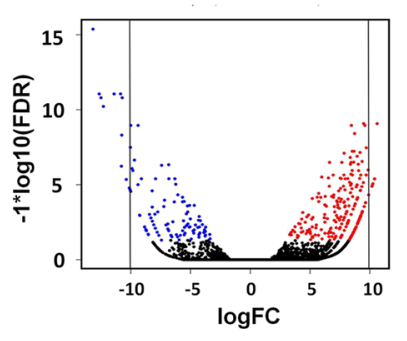

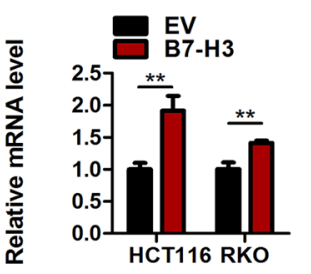

C

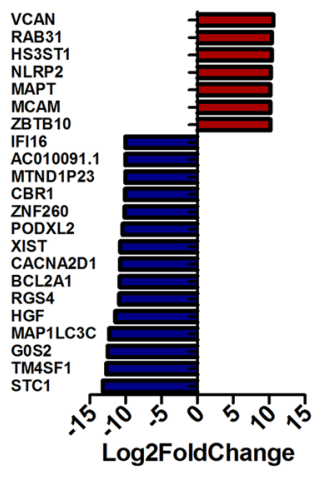

G
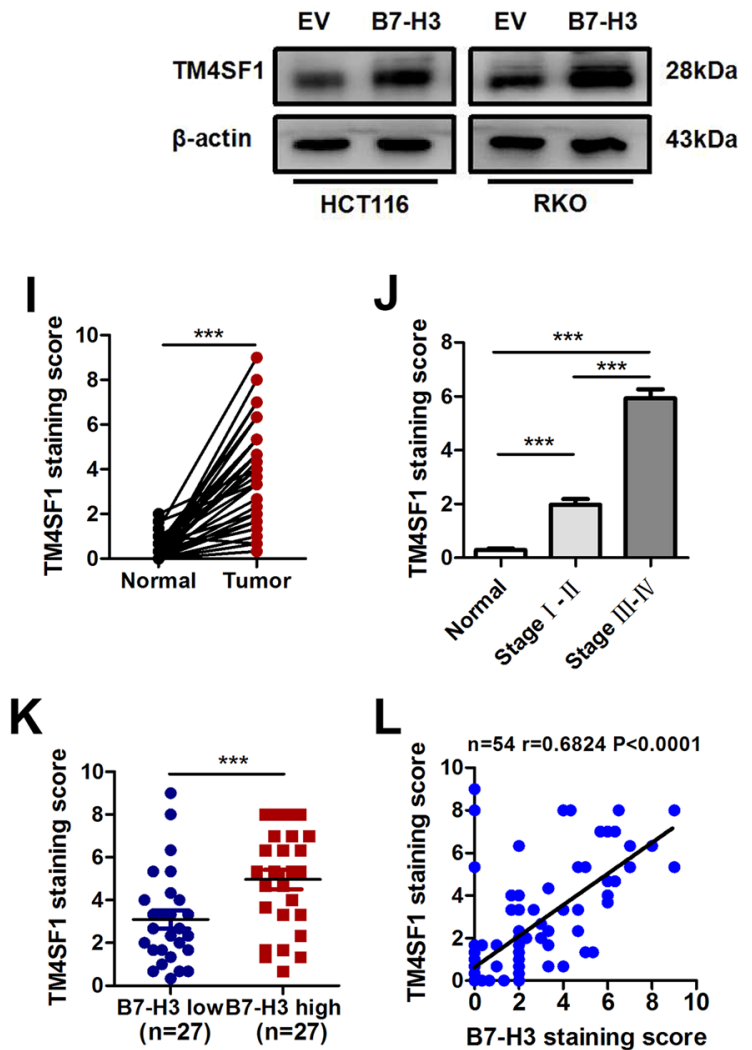

Fig. 3 B7-H3 promotes the expression of TM4SF1 in DOX-induced senescence. A RNA sequencing (RNA-seq) analysis showed the number of differentially expressed genes (DEGs) (139 downregulated and 300 upregulated) in sh-NC cells or shB7-H3 RKO cells with DOX treatment. B Image of RNA-Seq volcano plot showing logarithm of fold change in DEGs. C DEGs with logarithm of fold change $(|\operatorname{logFC}|)>10$ and $P$-value $<0.05$ were identified. D The mRNA expression of TM4SF1 in sh-NC cells or shB7-H3 CRC cells treated with DOX. E The mRNA expression of TM4SF1 in EV cells or B7-H3 CRC cells treated with DOX. F Western blot analysis of TM4SF1 and p21 in sh-NC cells or shB7-H3 CRC cells treated with DOX. $\beta$-Actin served as a loading control. G Western blot analysis of TM4SF1 and p21 in EV cells or B7-H3 CRC cells treated with DOX. $\beta$-Actin served as a loading control. H Representative images of $\mathrm{HC}$ for B7-H3 and TM4SF1 in CRC tissues and matched normal tissues from 54 clinical CRC patients. Scale bar, $100 \mu$ m. I TM4SF1 protein expression based on the staining index of CRC specimens and matched normal tissues. J TM4SF1 protein expression based on staining index in CRC specimens at different clinical stages. K TM4SF1 protein expression is shown for patients stratified into B7-H3 low (<median value) and B7-H3 high (>median value) groups. L Correlation analysis of the staining scores (protein expression levels) of B7-H3 and TM4SF1 in human CRC specimens $(n=54)$. The correlation coefficient $(r)$ is shown. The data represent the means \pm SEM. ${ }^{* *} P<0.01 ;{ }^{* * *} P<0.001$. 

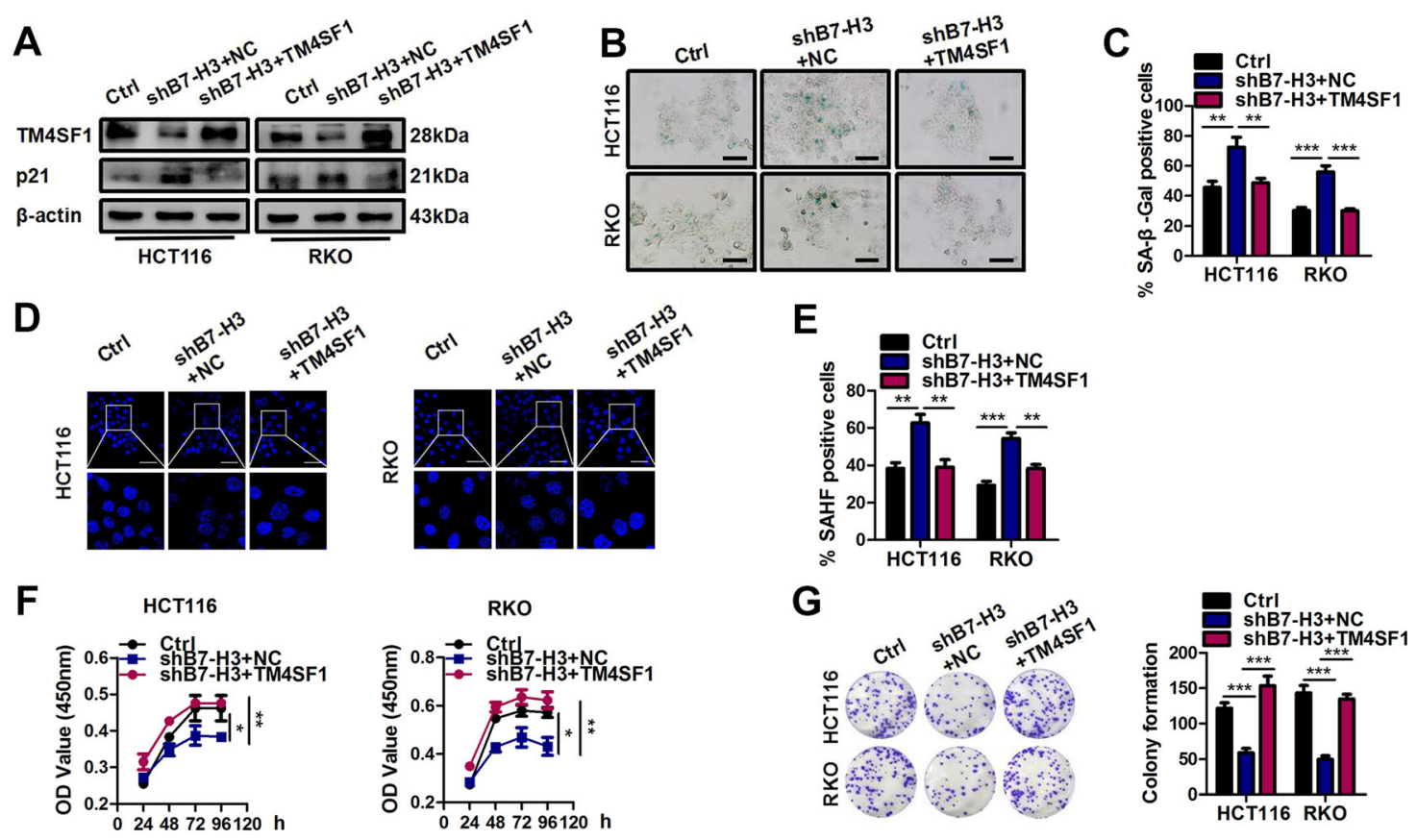

H
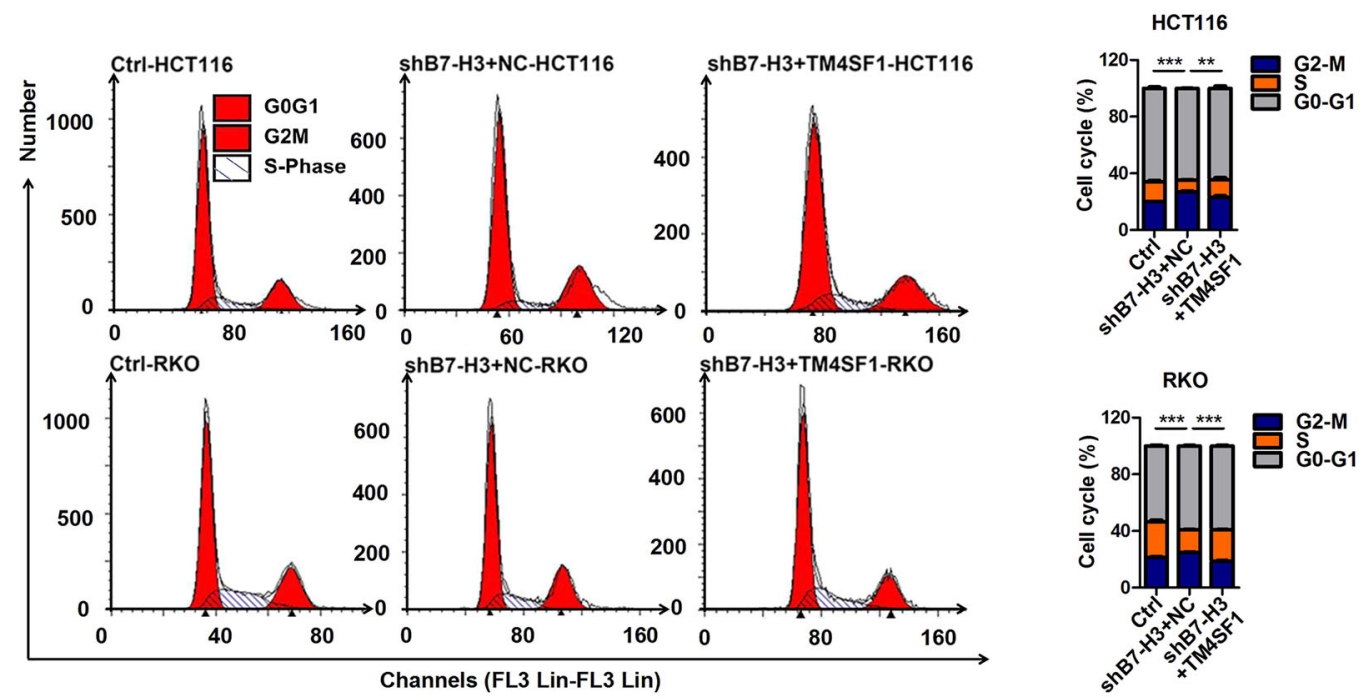

Fig. 4 TM4SF1 is involved in B7-H3-associated resistance to DOX-induced senescence. A Western blot analysis of TM4SF1, and p21 in control cells or shB7-H3 CRC cells after treatment with TM4SF1 overexpression vectors and DOX. $\beta$-Actin served as a loading control. B, C SA- $\beta$-Gal activity of control cells or shB7-H3 CRC cells after treatment with TM4SF1 overexpression vectors and DOX was examined. Scale bar, $100 \mu \mathrm{m}$. One representative image from three reproducible experiments is shown. The percentages of SA- $\beta$-gal-positive cells are shown in the bar graph. D, E SAHF activity of control cells or shB7-H3 CRC cells after treatment with TM4SF1 overexpression vectors and DOX was examined. Scale bar, $50 \mu \mathrm{m}$. One representative image from three replicate experiments is shown. The percentages of SAHF-positive cells are shown in the bar graph. $\mathbf{F}$ The cell viability of control cells or shB7-H3 CRC cells with TM4SF1 overexpression vectors and DOX treatment after 24, 36, 48, and $96 \mathrm{~h}$ was examined by CCK-8 assays. G The colony formation of control cells or shB7-H3 CRC cells after treatment with TM4SF1 overexpression vectors and DOX was examined. One representative image from three reproducible experiments is shown. The number of colonies is shown in the bar graph. $\mathbf{H}$ Cell cycle analysis by PI staining in control cells or shB7-H3 CRC cells after treatment with TM4SF1 overexpression vectors and DOX was examined through flow cytometry. The data represent the means \pm SEM. ${ }^{*} P<0.05 ;{ }^{* *} P<0.01 ;{ }^{* * *} P<0.001$.

SAHF-positive cells (Fig. 5A, B), as well as the protein level of p21 in B7-H3-overexpressing CRC cells treated with low-dose DOX (Fig. 5C). Moreover, TM4SF1
siRNA-2 transfection abolished the decrease in cell growth arrest and G2/M phase arrest in B7-H3overexpressing CRC cells treated with low-dose DOX 

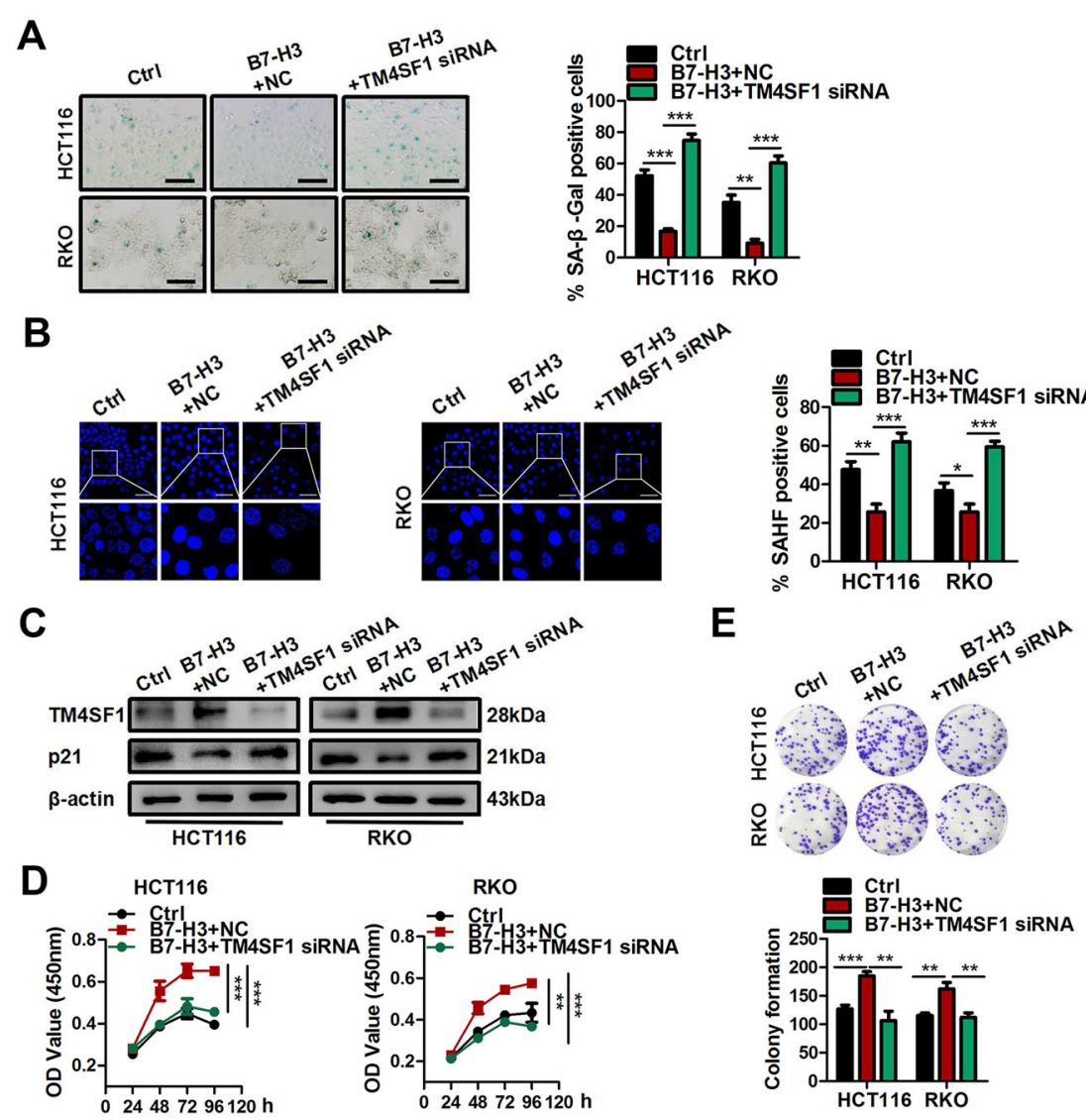

$\mathbf{F}$
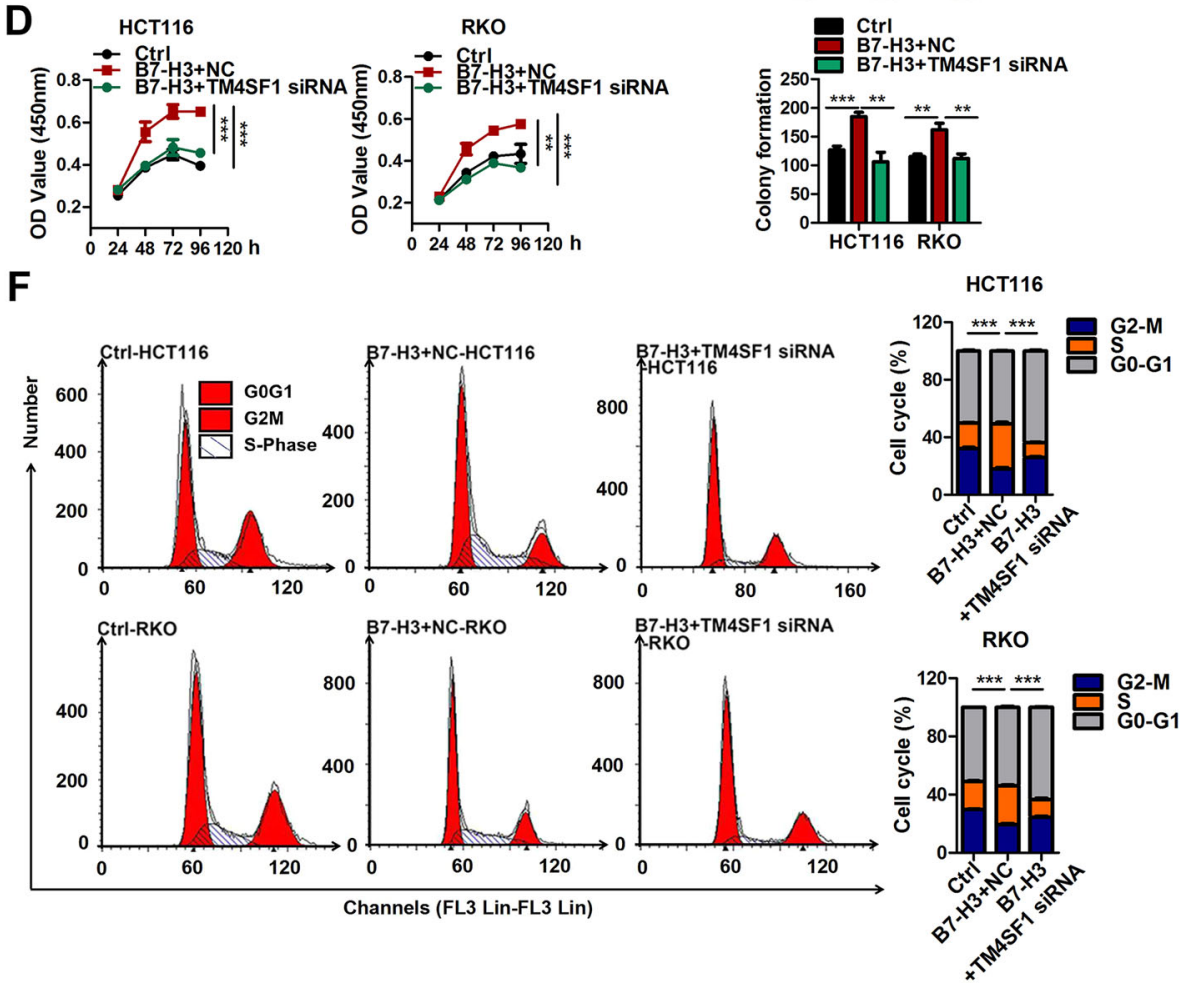

Fig. 5 Knockdown of TM4SF1 reverses B7-H3-associated resistance to DOX-induced senescence. A SA- $\beta$-Gal activity of control cells or B7-H3 CRC cells cotreated with TM4SF1 siRNA and DOX was examined. Scale bar, $100 \mu \mathrm{m}$. One representative image from three reproducible experiments is shown. The percentages of SA- $\beta$-gal-positive cells are shown in the bar graph. B SAHF activity of control cells or B7-H3 CRC cells cotreated with TM4SF1 siRNA and DOX was examined. Scale bar, $50 \mu \mathrm{m}$. One representative image from three replicate experiments. C Western blot analysis of TM4SF1 and p21 in control cells or B7-H3 CRC cells cotreated with TM4SF1 siRNA and DOX. $\beta$-Actin served as a loading control. D The cell viability of control cells or B7-H3 CRC cells cotreated with TM4SF1 siRNA and DOX after 24, 36, 48, and $96 \mathrm{~h}$ was examined by CCK-8 assays. E The colony formation of control cells or B7-H3 CRC cells with TM4SF1 siRNA and DOX cotreatment was examined. One representative image from three reproducible experiments is shown. The number of colonies is shown in the bar graph. $\mathbf{F}$ Cell cycle analysis by PI staining in control cells or B7-H3 CRC cells with TM4SF1 siRNA and DOX cotreatment was examined through flow cytometry. The data represent the mean \pm SEM. ${ }^{* * P}<0.01 ;{ }^{* *} P<0.001$. 
(Fig. 5D-F). These results illustrated that B7-H3 suppressed cellular senescence and senescence-like cell growth arrest via TM4SF1.

\section{B7-H3-mediated promotion of TM4SF1 expression and inhibition of cellular senescence is dependent on the AKT pathway in CRC}

Our previous studies showed that B7-H3 overexpression activated downstream signaling pathways, such as the AKT, NF-kB, and STAT3 pathways, in CRC cells $^{27}$. In the present study, Kyoto Encyclopedia of Genes and Genomes (KEGG) analysis showed that the AKT pathway was identified as a differentially enriched pathway in B7-H3 knockdown RKO cells treated with lowdose DOX (Supplementary Fig. S4A). Hence, we hypothesized that B7-H3 might promote TM4SF1 expression in low-dose DOX-induced senescent CRC cells via the AKT pathway. As shown in Supplementary Fig. S5B, treatment with the AKT inhibitor perifosine significantly reduced the mRNA level of TM4SF1 in low-dose DOXinduced senescent B7-H3-overexpressing HCT116 and RKO cells, while NF- $\mathrm{kB}$ inhibitor (BAY11-7082) or STAT3 inhibitor (cryptotanshinone) treatment had no effect on TM4SF1 mRNA expression. Moreover, perifosine administration obviously reduced the phosphorylation levels of AKT and the protein expression of TM4SF1 in low-dose DOX-induced senescent B7-H3overexpressing HCT116 and RKO cells (Fig. 6A), suggesting that $\mathrm{B} 7-\mathrm{H} 3$ promoted TM4SF1 expression in an AKT pathway-dependent manner. We then asked whether the AKT pathway was attributed to B7-H3-regulated cellular senescence in CRC cells. Perifosine treatment led to a significant increase in the percentages of SA- $\beta$-galand SAHF-positive cells (Fig. 6B-E), as well as the protein level of $\mathrm{p} 21$, in $\mathrm{B} 7-\mathrm{H} 3$-overexpressing CRC cells treated with low-dose DOX (Fig. 6A). Furthermore, perifosine administration abolished the decrease in cell growth arrest and G2/M phase arrest in B7-H3-overexpressing CRC cells treated with low-dose DOX (Fig. 6F-H).

\section{SIRT1 is required for B7-H3/TM4SF1 axis-regulated cellular senescence}

Sirtuin 1 (SIRT1), a key member of the deacetylase Sirtuin family, has been shown to play vital roles in modulating cellular senescence and aging ${ }^{34}$. In addition, TM4SF1 was involved in apoptosis, the cell cycle, and ROS metabolism of bladder cancer cells through the PPAR $\gamma$-SIRT1 feedback loop ${ }^{35}$. Thus, it becomes indispensable to find the relationship between SIRT1 and the B7-H3/TM4SF1 axis in low-dose DOX-induced cellular senescence. The western blot results showed that B7-H3 knockdown decreased SIRT1 protein expression, while TM4SF1 overexpression reversed the effect of B7-H3 knockdown on SIRT1 expression in low-dose DOX- induced senescent HCT116 and RKO cells (Supplementary Fig. S5A). Moreover, B7-H3 overexpression increased SIRT1 protein expression, while TM4SF1 knockdown abolished the effect of B7-H3 overexpression on SIRT1 expression in low-dose DOX-induced senescent CRC cells (Supplementary Fig. S5B). These results indicated that the B7-H3/TM4SF1 axis was able to regulate SIRT1 expression in low-dose DOX-induced senescent CRC cells. To further investigate the roles of SIRT1 in B7-H3/ TM4SF1 axis-regulated cellular senescence, we used a commercial SIRT1 siRNA and a TM4SF1 overexpression vector to cotransfect HCT116 and RKO cells. The results showed that TM4SF1 overexpression increased SIRT1 protein expression, while SIRT1 knockdown abolished the effect of TM4SF1 overexpression on SIRT1 expression in low-dose DOX-induced senescent shB7-H3 CRC cells (Fig. 7A). Moreover, SIRT1 knockdown reversed the effect of TM4SF1 overexpression on the percentages of SA$\beta$-gal- and SAHF-positive cells (Fig. 7B-E), as well as the protein level of p21 in B7-H3 knockdown CRC cells treated with low-dose DOX (Fig. 7A). In addition, SIRT1 knockdown abolished the decrease in cell growth arrest and G2/M phase arrest induced by TM4SF1 overexpression in $\mathrm{B} 7-\mathrm{H} 3$ knockdown CRC cells treated with low-dose DOX (Fig. 7F-H). The above results indicate that the B7-H3/TM4SF1 axis modulates low-dose DOXinduced cellular senescence via SIRT1.

\section{B7-H3 inhibited DOX-induced cellular senescence in vivo}

To verify the role of B7-H3 in DOX-induced cellular senescence in vivo, we first established a low-dose DOX-induced cell senescence model in nude mice. We found that low-dose DOX treatment $(4 \mathrm{mg} / \mathrm{kg}) \mathrm{sig}$ nificantly reduced the sizes and weight of tumors (Supplementary Fig. S6A-C). More importantly, treatment with low-dose DOX increased the percentage of SA- $\beta$-gal staining and the expression of p21 in tumors (Supplementary Fig. S6D, E). These results suggested that treatment with low-dose DOX induced cellular senescence in vivo.

B7-H3 knockdown significantly decreased HCT116 tumor growth after treatment with low-dose DOX, evidenced by the tumor sizes, images, and weight (Fig. $8 \mathrm{~A}-\mathrm{C})$. B7-H3 overexpression had the opposite effect (Supplementary Fig. S7A-C). The percentage of SA- $\beta$-gal staining and the expression of p21 were obviously increased in shB7-H3 HCT116 tumors treated with lowdose DOX (Fig. 8D-H). Conversely, decreased percentage of SA- $\beta$-gal staining and p21 expression was observed in B7-H3 HCT116 tumors treated with low-dose DOX (Supplementary Fig. S7D-H). Moreover, B7-H3 knockdown significantly decreased, while B7-H3 overexpression markedly increased the expression of TM4SF1 and SIRT1 in HCT116 tumors treated with low-dose DOX (Fig. 8I, J 


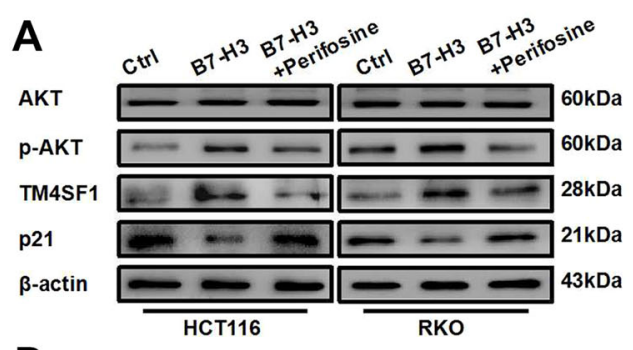

D

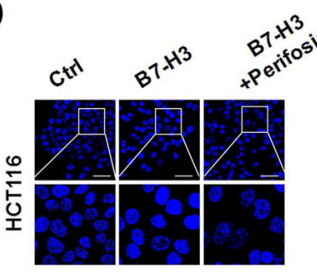

F

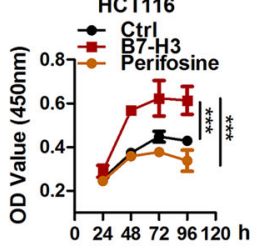

B

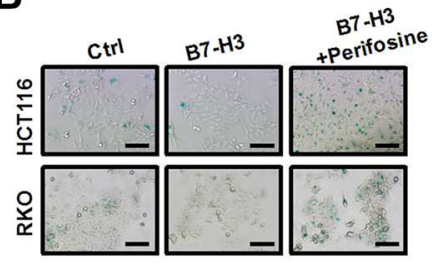

E

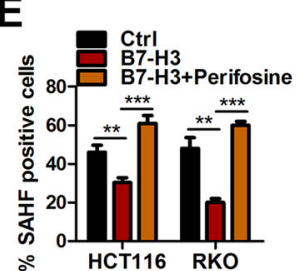

C

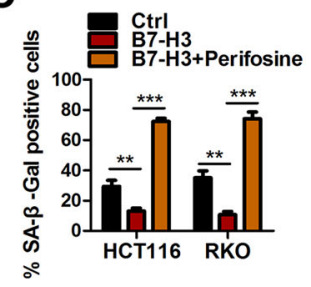

○
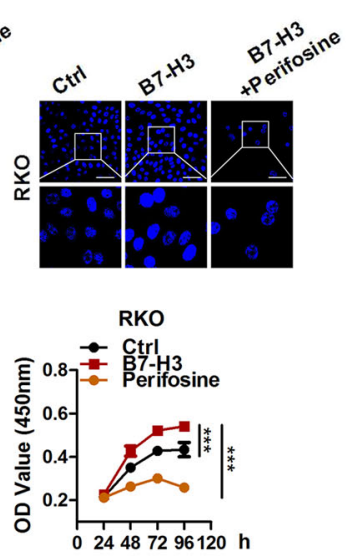

G

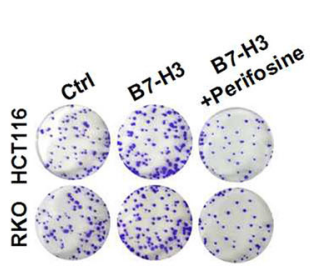

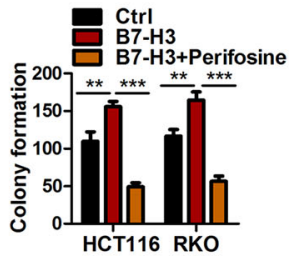

HCT116 RKO

H
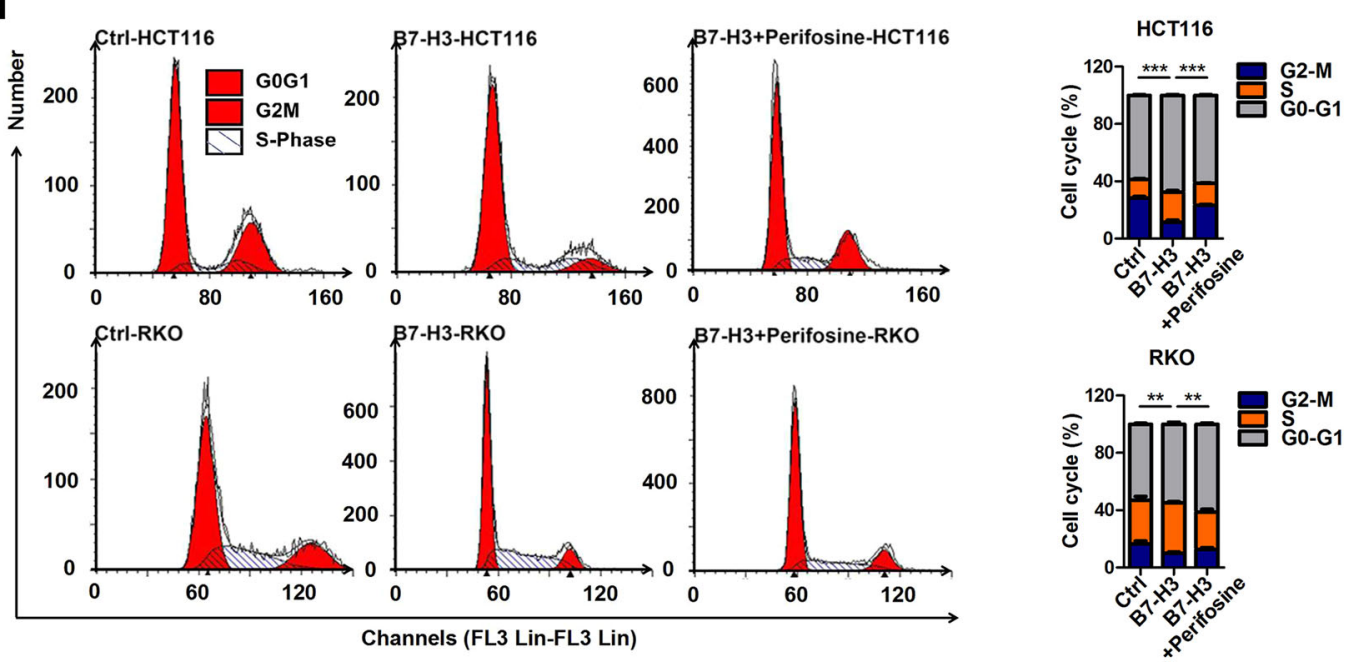

Fig. 6 B7-H3 promotes TM4SF1 expression and inhibits cellular senescence dependent on the AKT pathway in CRC. A Western blot analysis of AKT, p-AKT, TM4SF1 and p21 in control cells or B7-H3 CRC cells cotreated with perifosine and DOX. $\beta$-Actin served as a loading control. B, C SA$\beta$-Gal activity of control cells or B7-H3 CRC cells cotreated with perifosine and DOX was examined in CRC cells. Scale bar, $100 \mu \mathrm{m}$. One representative image from three reproducible experiments is shown. The percentages of SA- $\beta$-gal-positive cells are shown in the bar graph. D, E SAHF activity of control cells or B7-H3 CRC cells cotreated with perifosine and DOX was examined. Scale bar, $50 \mu \mathrm{m}$. One representative image from three replicate experiments. F The cell viability of control cells or B7-H3 CRC cells cotreated with perifosine and DOX after 24,36, 48, and $96 \mathrm{~h}$ was examined by CCK8 assays. G Colony formation assay in control cells or B7-H3 CRC cells cotreated with perifosine and DOX. One representative image from three reproducible experiments is shown. The number of colonies is shown in the bar graph. $\mathbf{H}$ Cell cycle analysis by PI staining in control cells or B7-H3 CRC cells with perifosine and DOX cotreatment was examined through flow cytometry. The data represent the mean \pm SEM. ${ }^{* *} P<0.01 ;{ }^{* * *} P<0.001$.

and Supplementary Fig. S7I-J). Taken together, we illustrated that B7-H3 could inhibit DOX-induced cellular senescence of CRC cells in vivo by regulating TM4ST1/ SIRT1 expression.

\section{Discussion}

Cellular senescence is considered as a kind of stress response induced by a variety of intrinsic and extrinsic insults $^{36}$. Nowadays, cellular senescence, especially 
A

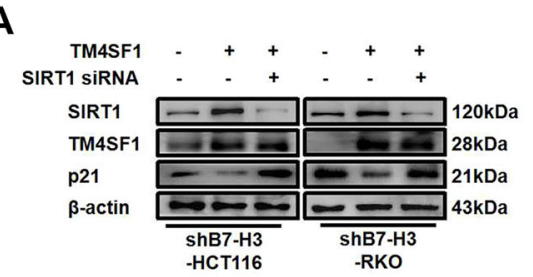

D

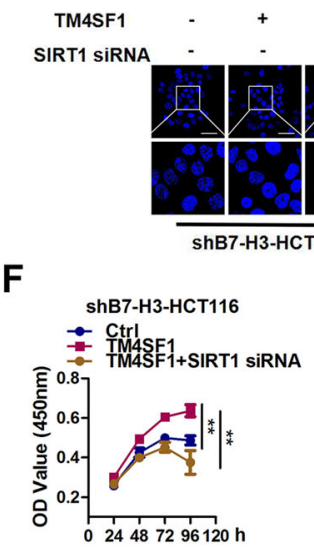

H
B

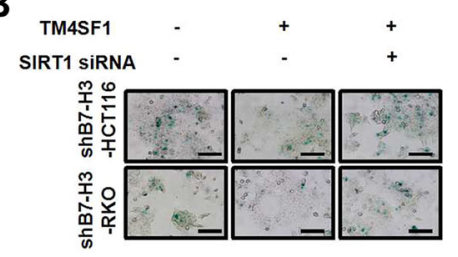

$\mathbf{E}$
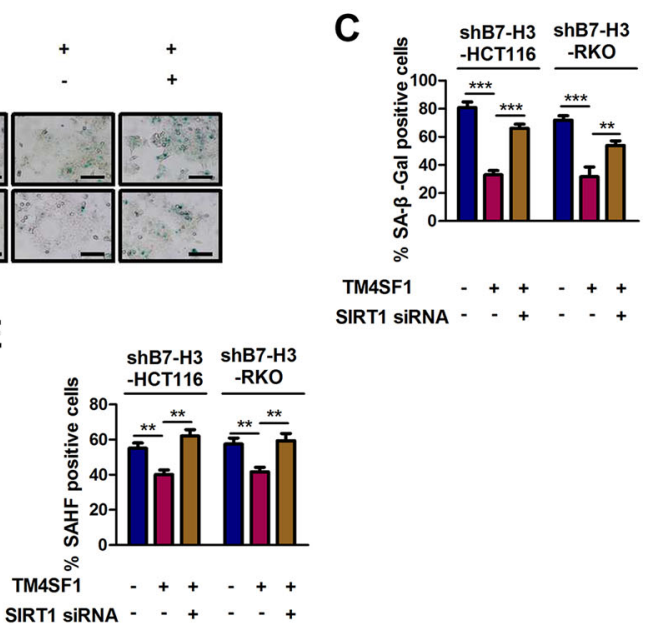

G

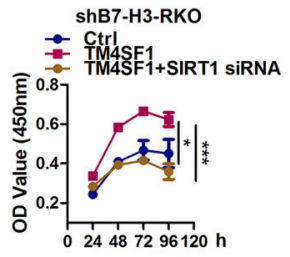

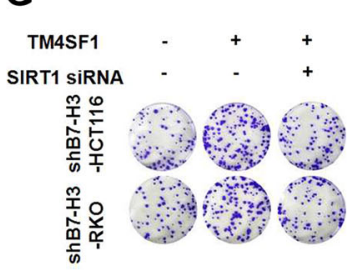

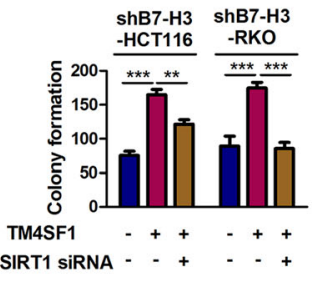

SIRT1 SiRNA - + + - +
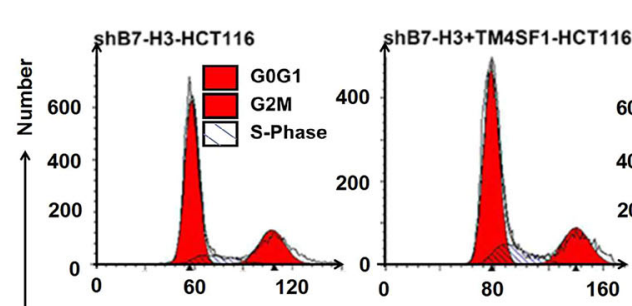

ShB7-H3+TM4SF1
+SIRT1 SiRNA-HCT116
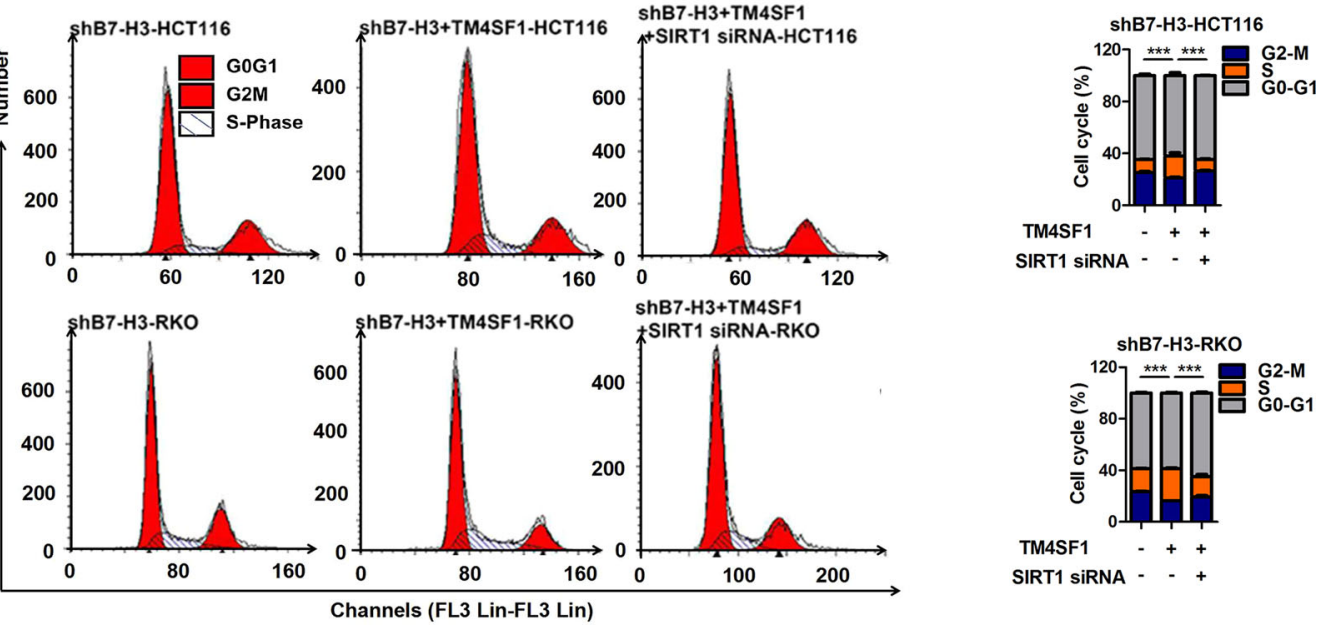

TM4SF1 SIRT1 SiRNA - - +

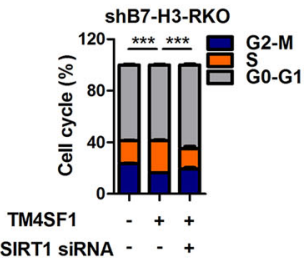

Fig. 7 SIRT1 is required for the resistance to B7-H3-mediated senescence. A Western blot analysis of SIRT1, TM4SF1, and p21 in DOX-treated shB7-H3 CRC cells cotreated with TM4SF1 overexpression vectors and SIRT1 siRNA. $\beta$-Actin served as a loading control. B, C SA- $\beta$-Gal activity of DOXtreated shB7-H3 CRC cells with TM4SF1 overexpression vectors and SIRT1 siRNA cotreatment was examined. Scale bar, $100 \mu \mathrm{m}$. One representative image from three reproducible experiments is shown. The percentages of SA- $\beta$-gal-positive cells are shown in the bar graph. D, E SAHF activity of DOX-treated shB7-H3 CRC cells with TM4SF1 overexpression vectors and SIRT1 siRNA cotreatment was examined. Scale bar, $50 \mu \mathrm{m}$. One representative image from three replicate experiments. $\mathbf{F}$ The cell viability of control cells or DOX-treated shB7-H3 CRC cells cotreated with TM4SF1 overexpression vectors and SIRT1 siRNA after 24, 36, 48, and $96 \mathrm{~h}$ was examined by CCK-8 assays. G The colony formation of control cells or DOXtreated shB7-H3 CRC cells with TM4SF1 overexpression vectors and SIRT1 siRNA cotreatment was examined. One representative image from three reproducible experiments is shown. The number of colonies is shown in the bar graph. $\mathbf{H}$ Cell cycle analysis by PI staining in control cells or DOXtreated shB7-H3 CRC cells with TM4SF1 overexpression vectors and SIRT1 siRNA cotreatment was examined through flow cytometry. The data represent the means \pm SEM. ${ }^{*} P<0.05 ;{ }^{*} P<0.01 ;{ }^{* *} P<0.001$.

therapy-induced senescence (TIS), has been recognized as a new weapon for cancer therapy ${ }^{37}$. Hence, increasing evidence has indicated the underlying molecular mechanisms of this process in cancer cells. For instance, EZH2 mediates lidamycin-induced cellular senescence in a p21-dependent manner in CRC cells ${ }^{38}$. AP4, the 


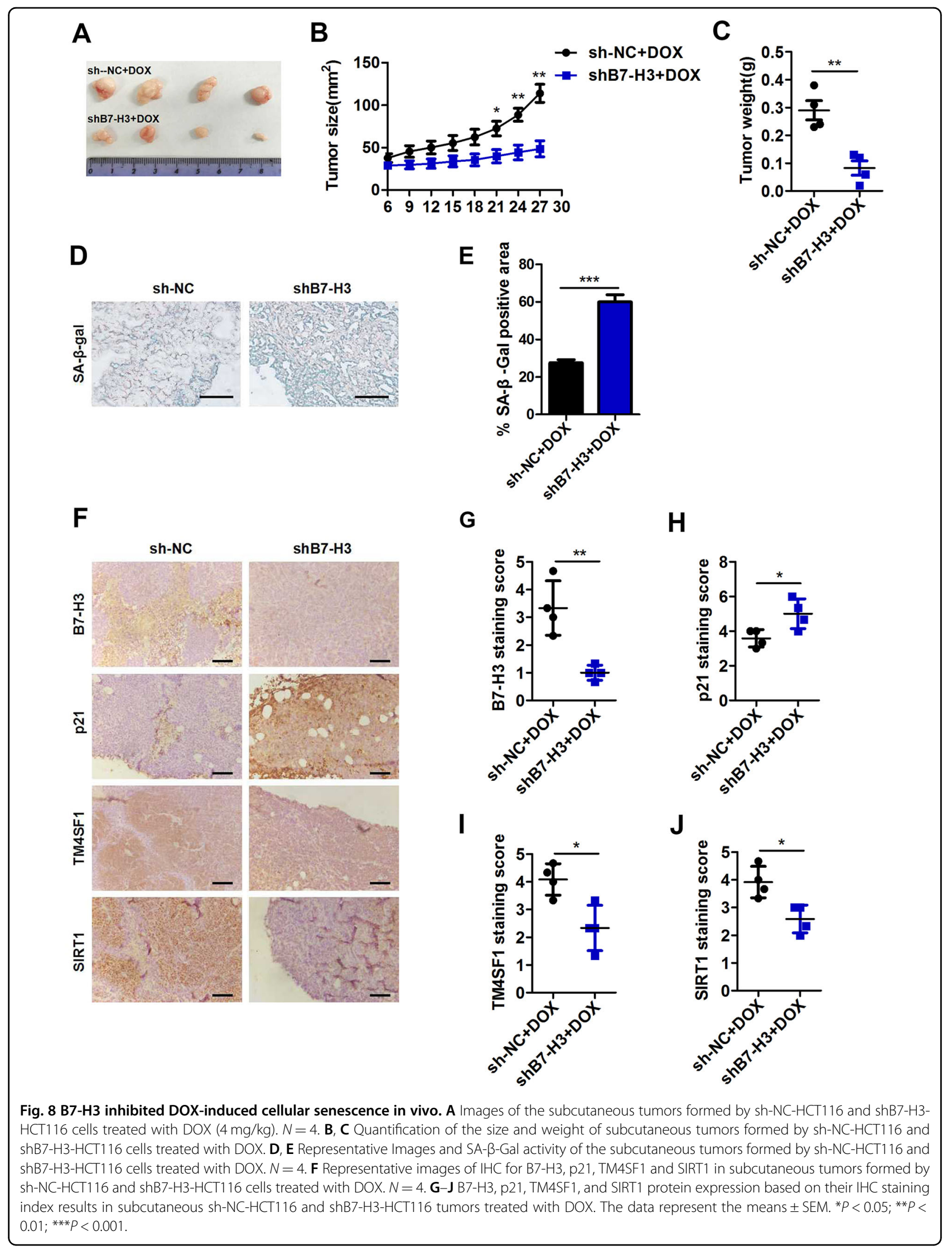


chromatin remodeling enzyme, downregulates p16 and p21 to suppress senescence ${ }^{39}$. Zhang and coworkers found that inhibition of TAZ contributes to radiationinduced senescence and growth arrest in glioma cells ${ }^{40}$. In this study, we induced cellular senescence in CRC cells by a low dose of DOX and demonstrated that B7-H3 knockdown promoted, while $\mathrm{B} 7-\mathrm{H} 3$ overexpression inhibited, cellular senescence induced by a low dose of DOX. Importantly, B7-H3 knockdown dramatically enhanced the growth arrest of CRC cells after low-dose DOX treatment, but the overexpression of B7-H3 had the opposite effect. In addition, B7-H3 mediated cellular senescence induced by a low dose of DOX in vivo. These results suggest that $\mathrm{B} 7-\mathrm{H} 3$ is an important suppressor of low-dose DOX-induced senescence in CRC. Apart from therapy-induced senescence, replicative senescence, oncogene-induced senescence, and PTEN-loss induced cellular senescence also exert important role in tumor progression and therapy ${ }^{41,42}$. At the current stage we could not clarify the precise functions of $\mathrm{B} 7-\mathrm{H} 3$ in regulating replicative senescence, oncogene-induced senescence, and PTEN-loss induced cellular senescence in CRC. Further investigations are required to answer this question.

Recent evidence indicated that cellular senescence acts as a double-edged sword during tumorigenesis because it promotes tumorigenesis mainly through the SASP apart from its tumor-suppressing functions ${ }^{11}$. Thus far, eliminating senescent cells or suppressing SASP has received wide attention. Athena et al. showed that PTBP1 depletion prevented the protumorigenic effects of SASP and inhibited inflammation-driven cancer $^{43}$. Apigenin mediated suppression of the SASP and reduced the aggressive phenotype of human breast cancer cells ${ }^{44}$. It has been reported that B7-H3 is involved in the production of cytokines such as IL-6, IL8 , and VEGF in cancers ${ }^{27,45,46}$. Therefore, we surmised that B7-H3 might play important roles in regulating the SASP in senescent CRC cells induced by a low dose of DOX. Unfortunately, we did not investigate the effect of B7-H3 on SASP in the present study. It would be interesting to further elaborate the functions of B7-H3 in modulating SASP in CRC.

The most widely studied pathways regulating cellular senescence are $\mathrm{p} 53 / \mathrm{p} 21^{\mathrm{cip} 1}$ and/or $\mathrm{p} 16^{\mathrm{INK} 4 \mathrm{~A}} / \mathrm{Rb}$ tumor suppressor pathways ${ }^{47-49}$. DNA damage and/or the DNAdamage response (DDR) are seemingly the two crucial parts controlling these two pathways ${ }^{50,51}$. It has been reported that p53 regulates the expression of a great deal of target genes involved in senescence ${ }^{52}$. Herein, we observed that both $\mathrm{p} 53$ and $\mathrm{p} 21$ protein expression were increased in HCT116 and RKO cells after treatment with a low dose of DOX. However, B7-H3 alteration regulated p21 expression but did not affect the expression of p53 in low-dose DOX-induced senescent CRC cells. These results indicated that $\mathrm{B} 7-\mathrm{H} 3$ regulated $\mathrm{p} 21$ expression and low-dose DOX-induced cellular senescence in a p53independent manner. In line with our results, many novel regulators, such as ARF, TRIB2, and SETD8, are involved in p53-independent cellular senescence ${ }^{16,53,54}$.

To extensively explore the mechanisms responsible for B7-H3-mediated regulation of cellular senescence in CRC cells, we performed RNA-seq analysis to identify the DEGs in shB7-H3 RKO cells treated with low-dose DOX and found that TM4SF1 was one of the highest rated DEGs. TM4SF1, a member of the TM4SF protein family, is identified as an oncogene and regulates proliferation, metastasis, and invasion in various cancers, including $\mathrm{CRC}^{55}$. Moreover, it has been reported that TM4SF1 is one of the top 10 upregulated genes and can act as marker of poor prognosis in $\mathrm{CRC}^{56}$. In addition, there is evidence that TM4SF1 is also an important regulator of senescence $^{57,58}$. The expression of TM4SF1 was increased in senescent human mesenchymal stem cells, which seemed to affect cellular senescence ${ }^{57}$. Moreover, TM4SF1 regulated endothelial cell functions, including filopodia formation, cell mobility, cytokinesis, cellular senescence, and tumor angiogenesis ${ }^{58}$. In the current work, we verified both the mRNA and protein expression of TM4SF1 in low-dose DOX-induced senescent CRC cells, which was significantly downregulated in B7-H3 knockdown cells but upregulated in B7-H3-overexpressing cells. Furthermore, TM4SF1 expression was positively correlated with B7-H3 expression in human CRC tissues. More importantly, silencing of TM4SF1 obviously abated B7-H3mediated resistance to cell senescence, while the overexpression of TM4SF1 substantially alleviated the degree of senescence in B7-H3 knockdown CRC cells. Thus, we have shown that $\mathrm{B} 7-\mathrm{H} 3$ enhances the resistance to lowdose DOX-induced senescence of CRC cells via TM4SF1.

AKT is known to be one of the important central nodes in different signaling pathways during tumorigenesis ${ }^{59}$. Frequent hyperactivation of AKT kinases has widely been found in various human solid tumors. Inhibiting the AKT pathway is closely related to changing several biological characteristics in cancers, such as cell proliferation, cell cycle distribution, and cellular senescence ${ }^{60,61}$. Hirose and coworkers showed that AKT activation could suppress temozolomide-induced mitotic catastrophe and cellular senescence in glioblastoma cells ${ }^{62}$. In the present study, KEGG pathway enrichment analysis indicated that DEGs in B7-H3 knockdown RKO cells treated with low-dose DOX were clearly enriched in the AKT pathway. Previous studies have shown that TM4SF1 is well known for its oncogenic functions in human cancers via the AKT pathway $^{33,63}$. Additionally, we previously demonstrated that B7-H3 overexpression could activate the AKT pathway in CRC cells ${ }^{27}$. Therefore, we hypothesized that B7H3 might promote TM4SF1 expression in low-dose 
DOX-induced senescent CRC cells via the AKT pathway. As expected, our Western blot assay indicated that perifosine, an AKT inhibitor, markedly reduced the phosphorylation levels of AKT and the expression of TM4SF1 in lowdose DOX-induced senescent B7-H3-overexpressing CRC cells, providing evidence that $\mathrm{B} 7-\mathrm{H} 3$ modulated TM4SF1 expression in senescent CRC cells via the AKT signaling pathway. Importantly, we observed that perifosine treatment led to a significant decrease in B7-H3-mediated resistance to cellular senescence. This suggests that $\mathrm{B} 7-\mathrm{H} 3$ is involved in low-dose DOX-induced senescence of CRC cells via the AKT/TM4SF1 pathway.

SIRT1 is categorized as a class III histone deacetylase and plays a role in a wide range of cellular functions, such as metabolism, differentiation, and senescence ${ }^{64-66}$. Importantly, SIRT1 overexpression has been discovered in many cancers, including $\mathrm{CRC}^{67}$. Moreover, the expression level of TM4SF1 obviously influenced the expression of SIRT1 in senescent models, as reported previously ${ }^{35}$. It has been confirmed that p21, an important cellular senescence marker, can be suppressed by SIRT1 in a p53-dependent and independent manner ${ }^{68,69}$. These findings led us to postulate that SIRT1 is of great importance in B7-H3-mediated resistance to cellular senescence through the AKT/TM4SF1 axis. Consistent with the change in TM4SF1 expression, SIRT1 expression was reduced in B7-H3 knockdown cells but increased in B7-H3-overexpressing cells. Moreover, TM4SF1 overexpression reversed the decrease in SIRT1 protein expression caused by $\mathrm{B} 7-\mathrm{H} 3$ knockdown in lowdose DOX-induced senescent CRC cells. Consistently, TM4SF1 knockdown abolished the effect of B7-H3 overexpression on SIRT1 expression in low-dose DOX-induced senescent CRC cells. More importantly, SIRT1 knockdown abolished B7-H3/TM4SF1 axis-mediated resistance to cell senescence. These results firmly indicate that B7-H3 confers resistance to low-dose DOX-induced senescence mainly through the AKT/TM4SF1/SIRT1 pathway. Although our results indicated that B7-H3/AKT/TM4SF1/ SIRT1 axis is able to regulate p21 expression in low-dose DOX-induced senescent CRC cells, the precise way of this axis to $\mathrm{p} 21$ expression is still unclear.

Nowadays, several blocking antibodies against B7-H3 antibodies have been developed and used for the therapy of solid tumors. $8 \mathrm{H} 9$, a monoclonal antibody specific for 4Ig-B7-H3, has a potent effect for patients with B7-H3 positive tumors in radioimmunotherapy ${ }^{70}$. Besides, humanized monoclonal antibodies targeting B7-H3, enoblituzumab, are under clinical investigation for therapeutic use in human prostate cancer ${ }^{71}$. Given our results that B7-H3 played important roles in low-dose DOXinduced senescence of CRC cells in vitro and in vivo, we speculated that combination B7-H3 blockade therapy and TIS therapy based on low-dose DOX would become a promising strategy for CRC patients.
Our findings, for the first time, indicate that the high expression of $\mathrm{B} 7-\mathrm{H} 3$ exacerbates the resistance to low-dose DOX-induced senescence through the AKT/TM4SF1/SIRT1 pathway. B7-H3 may act not only as a biomarker for predicting the response to low-dose DOX-induced senescence before it becomes clinically apparent but also as a target for potential paths for the development clinical interventions, which may improve the efficiency of TIS in the future.

\section{Acknowledgements \\ Not applicable}

\section{Author details}

'Jiangsu Institute of Clinical Immunology, The First Affiliated Hospital of Soochow University, 708 Renmin Road, Suzhou, China. ${ }^{2}$ Department of Gastroenterology, The First Affiliated Hospital of Soochow University, 188 Shizi Road, Suzhou, China. ${ }^{3}$ Jiangsu Key Laboratory of Clinical Immunology, Soochow University, 708 Renmin Road, Suzhou, China. ${ }^{4}$ Suzhou Key Laboratory for Tumor Immunology of Digestive Tract, The First Affiliated Hospital of Soochow University, 708 Renmin Road, Suzhou, China. ${ }^{5}$ Department of Oncology, The First Affiliated Hospital of Soochow University, 188 Shizi Road, Suzhou, China. ${ }^{6}$ Jiangsu Key Laboratory of Gastrointestinal Tumor Immunology, The First Affiliated Hospital of Soochow University, 708 Renmin Road, Suzhou, China

\section{Author contributions}

R.Q.W. performed the experiments. R.Q.W., Y.C.M., and L.Q.S. analyzed the data and wrote the paper. T.G.S. and W.C.C. are the corresponding authors and they designed the research. S.H.Z. and S.H.X. performed sample collection and clinical evaluation. H.Y.W. provided guidance on experimental technology. W.C.C., T.G.S., X.G.Z., and G.B.Z. revised the paper critically for important intellectual content. All authors read and approved the final manuscript.

\section{Funding}

This work was supported by the National Natural Science Foundation of China (82073156, 81802843); Suzhou Science \& Technology plan project (SYS2019035); Postgraduate Research \& Practice Innovation Program of Jiangsu Province (KYCX20_2689).

\section{Ethics statement}

This study was approved by the Ethics Committee of The First Affiliated Hospital of Soochow University (approval no. 2020084), and informed consent was obtained from the patients. The animal assay was approved by the Institutional Animal Care and Use Committee of Soochow University (Suzhou, (hina).

\section{Conflict of interest}

The authors declare no competing interests.

\section{Publisher's note}

Springer Nature remains neutral with regard to jurisdictional claims in published maps and institutional affiliations.

Supplementary information The online version contains supplementary material available at https://doi.org/10.1038/s41419-021-03736-2.

Received: 6 February 2021 Revised: 19 April 2021 Accepted: 19 April 2021 Published online: 06 May 2021

\footnotetext{
References

1. Siegel, R. L., Miller, K. D. \& Jemal, A. Cancer statistics, 2020. CA Cancer J. Clin. 70 7-30 (2020).

2. Stein, A., Atanackovic, D. \& Bokemeyer, C. Current standards and new trends in the primary treatment of colorectal cancer. Eur. J. Cancer. 47, S312-S314 (2011).
} 
3. Carvalho, C. et al. Doxorubicin: the good, the bad and the ugly effect. Curr. Med Chem. 16, 3267-3285 (2009).

4. J, Zhu, R. et al. Knockdown of long non-coding RNA XIST inhibited doxorubicin resistance in colorectal cancer by upregulation of miR-124 and downregulation of SGK1. Cell. Physiol. Biochem. 51, 113-128 (2018).

5. Ma, K. et al. Nuclear accumulation of yes-associated protein (YAP) maintains the survival of doxorubicin-induced senescent cells by promoting survivin expression. Cancer Lett. 375, 84-91 (2016).

6. Rebbaa, A., Zheng, X., Chou, P. M. \& Mirkin, B. L. Caspase inhibition switches doxorubicin-induced apoptosis to senescence. Oncogene 22, 2805-2811 (2003).

7. Bai, J. et al. Inhibition enhancer of zeste homologue 2 promotes senescence and apoptosis induced by doxorubicin in p53 mutant gastric cancer cells. Cell Prolif. 47, 211-218 (2014).

8. Acosta, J. C. \& Gil, J. Senescence: a new weapon for cancer therapy. Trends Cell Biol. 22, 211-219 (2012).

9. Campisi, J. Aging, cellular senescence, and cancer. Annu Rev. Physiol. 75, 685-705 (2013).

10. Muñoz-Espín, D. \& Serrano, M. Cellular senescence: from physiology to pathology. Nat. Rev. Mol. cell Biol. 15, 482-496 (2014).

11. Coppé, J. P., Desprez, P. Y., Krtolica, A. \& Campisi, J. The senescence-associated secretory phenotype: the dark side of tumor suppression. Annu Rev. Pathol. 5, 99-118 (2010).

12. Lan, L. et al. Shp2 signaling suppresses senescence in PyMT-induced mammary gland cancer in mice. EMBO J. 34, 1493-1508 (2015).

13. Kim, Y. H. \& Park, T. J. Cellular senescence in cancer. BMB Rep. 52, 42-46 (2019).

14. Lee, S. \& Lee, J. S. Cellular senescence: a promising strategy for cancer therapy. BMB Rep. 52, 35-41 (2019).

15. Kovatcheva, M. et al. ATRX is a regulator of therapy induced senescence in human cells. Nat. Commun. 8, 386 (2017).

16. Hou, Z. et al. TRIB2 functions as novel oncogene in colorectal cancer by blocking cellular senescence through AP4/p21 signaling. Mol. Cancer 17, 172 (2018).

17. Wang, Z. et al. Nuclear receptor HNF4a performs a tumor suppressor function in prostate cancer via its induction of p21-driven cellular senescence. Oncogene 39, 1572-1589 (2020).

18. Chapoval, A. I. et al. B7-H3: a costimulatory molecule for T cell activation and IFN-gamma production. Nat. Immunol. 2, 269-274 (2001).

19. Ingebrigtsen, V. A. et al. B7-H3 expression in colorectal cancer: associations with clinicopathological parameters and patient outcome. BMC Cancer 14, 602 (2014).

20. Chen, Y. et al. The coexpression and clinical significance of costimulatory molecules B7-H1, B7-H3, and B7-H4 in human pancreatic cancer. Onco Targets Ther. 7, 1465-1472 (2014).

21. Zang, X. et al. B7-H3 and B7X are highly expressed in human prostate cancer and associated with disease spread and poor outcome. Proc. Natl Acad. Sci. USA 104, 19458-19463 (2007).

22. Zang, $\mathrm{X}$. et al. Tumor associated endothelial expression of $\mathrm{B} 7-\mathrm{H} 3$ predicts survival in ovarian carcinomas. Mod. Pathol. 23, 1104-1112 (2010).

23. Ni, L. \& Dong, C. New B7 family checkpoints in human cancers. Mol. Cancer Ther. 16, 1203-1211 (2017)

24. Nygren, M. K., Tekle, C., Ingebrigtsen, V. A. \& Fodstad, O. B7-H3 and its relevance in cancer; immunological and non-immunological perspectives. Front. Biosci. (Elite Ed.) 3, 989-993 (2011).

25. Kang, F. B. et al. B7-H3 promotes aggression and invasion of hepatocellular carcinoma by targeting epithelial-to-mesenchymal transition via JAK2/STAT3/ Slug signaling pathway. Cancer Cell Int. 15, 45 (2015).

26. Shi, T. et al. B7-H3 promotes aerobic glycolysis and chemoresistance in colorectal cancer cells by regulating HK2. Cell Death Dis. 10, 308 (2019).

27. Wang, $\mathrm{R}$. et al. B7-H3 promotes colorectal cancer angiogenesis through activating the NF-kB pathway to induce VEGFA expression. Cell Death Dis. 11, 55 (2020).

28. Liu, Z. et al. Immunoregulatory protein B7-H3 regulates cancer stem cell enrichment and drug resistance through MVP-mediated MEK activation. Oncogene 38, 88-102 (2019).

29. Flem-Karlsen, $\mathrm{K}$. et al. Immunoregulatory protein $\mathrm{B} 7-\mathrm{H} 3$ promotes growth and decreases sensitivity to therapy in metastatic melanoma cells. Pigment Cell melanoma Res. 30, 467-476 (2017).

30. Lehmann, B. D. et al. Senescence-associated exosome release from human prostate cancer cells. Cancer Res. 68, 7864-7871 (2008).

31. Park, Y. R. et al. MiRNA-206 suppresses PGE2-induced colorectal cancer cell proliferation, migration, and invasion by targetting TM4SF1. Biosci. Rep. 38, BSR20180664 (2018).
32. Cao, J. et al. TM4SF1 Promotes Gemcitabine Resistance of Pancreatic Cancer In Vitro and In Vivo. PLOS ONE 10, e0144969 (2015).

33. Ye, L. et al. Transmembrane-4 L-six family member-1 (TM4SF1) promotes nonsmall cell lung cancer proliferation, invasion and chemo-resistance through regulating the DDR1/Akt/ERK-mTOR axis. Respir. Res. 20, 106 (2019).

34. Hekmatimoghaddam, S., Firoozabadi, A. D., Zare-Khormizi, M. R. \& Pourrajab, F. Sirt1 and Parp1 as epigenome safeguards and microRNAs as SASP-associated signals, in cellular senescence and aging. Ageing Res. Rev. 40, 120-141 (2017).

35. Cao, R. et al. TM4SF1 regulates apoptosis, cell cycle and ROS metabolism via the PPARY-SIRT1 feedback loop in human bladder cancer cells. Cancer Lett. 414, 278-293 (2018).

36. Kuilman, T., Michaloglou, C., Mooi, W. J. \& Peeper, D. S. The essence of senescence. Genes Dev. 24, 2463-2479 (2010).

37. Acosta, J. C. \& Gil, J. Senescence: a new weapon for cancer therapy. Trends Cell Biol. 22, 211-219 (2012).

38. Sha, M. Q. et al. EZH2 mediates lidamycin-induced cellular senescence through regulating p21 expression in human colon cancer cells. Cell Death Dis. 7, e2486 (2016).

39. Jackstadt, R., Jung, P. \& Hermeking, H. AP4 directly downregulates p16 and p21 to suppress senescence and mediate transformation. Cell Death Dis. 4 e775 (2013).

40. Zhang, L. et al. Inhibition of TAZ contributes radiation-induced senescence and growth arrest in glioma cells. Oncogene 38, 2788-2799 (2019).

41. Nardella, C., Clohessy, J. G., Alimonti, A. \& Pandolfi, P. P. Pro-senescence therapy for cancer treatment. Nat. Rev. Cancer 11, 503-511 (2011).

42. Kovatcheva, M. et al. ATRX is a regulator of therapy induced senescence in human cells. Nat. Commun. 8, 386 (2017).

43. Georgilis, A. et al. PTBP1-mediated alternative splicing regulates the inflammatory secretome and the pro-tumorigenic effects of senescent cells. Cancer Cell 34, 85-102.e109 (2018).

44. Perrott, K. M., Wiley, C. D., Desprez, P. Y. \& Campisi, J. Apigenin suppresses the senescence-associated secretory phenotype and paracrine effects on breast cancer cells. GeroScience 39, 161-173 (2017).

45. Zhang, T., Jiang, B., Zou, S. T., Liu, F. \& Hua, D. Overexpression of B7-H3 augments anti-apoptosis of colorectal cancer cells by Jak2-STAT3. World J. Gastroenterol. 21, 1804-1813 (2015).

46. Xie, $\mathrm{C}$. et al. Soluble B7-H3 promotes the invasion and metastasis of pancreatic carcinoma cells through the TLR4/NF-KB pathway. Sci. Rep. 6, 27528 (2016).

47. Mijit, M., Caracciolo, V., Melillo, A., Amicarelli, F., \& Giordano, A. Giordano. Role of p53 in the regulation of cellular senescence. Biomolecules 10, 420 (2020).

48. Sharpless, N. E. \& Sherr, C. J. Forging a signature of in vivo senescence. Nat. Rev. Cancer 15, 397-408 (2015).

49. Chen, J. et al. Contribution of p16INK4a and p21CIP1 pathways to induction of premature senescence of human endothelial cells: permissive role of p53. Am. J. Physiol. Heart Circulatory Physiol. 290, H1575-H1586 (2006).

50. Malaquin, N., Carrier-Leclerc, A., Dessureault, M. \& Rodier, F. DDR-mediated crosstalk between DNA-damaged cells and their microenvironment. Front. Genet. 6, 94 (2015).

51. Vasileiou, P. V. S. et al. Mitochondrial homeostasis and cellular senescence. Cells 8, 686 (2019)

52. Fischer, M. Census and evaluation of p53 target genes. Oncogene $\mathbf{3 6}$ 3943-3956 (2017).

53. Chan, C. H., Gao, Y., Moten, A. \& Lin, H. K. Novel ARF/p53-independent senescence pathways in cancer repression. J. Mol. Med. 89, 857-867 (2011).

54. Shih, C. T. et al. The PPARY-SETD8 axis constitutes an epigenetic, p53independent checkpoint on p21-mediated cellular senescence. Aging Cell 16, 797-813 (2017)

55. Fan, C., Liu, N., Zheng, D., Du, J. \& Wang, K. MicroRNA-206 inhibits metastasis of triple-negative breast cancer by targeting transmembrane 4 L6 family member 1. Cancer Manag Res. 11, 6755-6764 (2019).

56. Martinez-Romero, J., Bueno-Fortes, S., Martín-Merino, M., de Molina, A. R., \& De Las Rivas, J. Survival marker genes of colorectal cancer derived from consistent transcriptomic profiling. BMC Genomics 19, 857 (2018).

57. Yoo, J. K., Choi, S. J. \& Kim, J. K. Expression profiles of subtracted mRNAs during cellular senescence in human mesenchymal stem cells derived from bone marrow. Exp. Gerontol. 48, 464-471 (2013).

58. Shih, S. C. et al. The L6 protein TM4SF1 is critical for endothelial cell function and tumor angiogenesis. Cancer Res. 69, 3272-3277 (2009).

59. Altomare, D. A. \& Testa, J. R. Perturbations of the AKT signaling pathway in human cancer. Oncogene 24, 7455-7464 (2005). 
60. Gao, Y. et al. Melatonin synergizes the chemotherapeutic effect of 5fluorouracil in colon cancer by suppressing PI3KAKT and NF-kB/iNOS signaling pathways. J. Pineal. Res. 62, e12380 (2017);

61. He, Q. et al. Dual inhibition of Akt and ERK signaling induces cell senescence in triple-negative breast cancer. Cancer Lett. 448, 94-104 (2019).

62. Hirose, Y., Katayama, M., Mirzoeva, O. K., Berger, M. S. \& Pieper, R. O. Akt activation suppresses Chk2-mediated, methylating agent-induced G2 arrest and protects from temozolomide-induced mitotic catastrophe and cellular senescence. Cancer Res. 65, 4861-4869 (2005).

63. Ma, Y. S. et al. miR-30 Family reduction maintains self-renewal and promotes tumorigenesis in NSCLC-initiating cells by targeting oncogene TM4SF1. Mol. Ther. 26, 2751-2765 (2018).

64. Michan, S. \& Sinclair, D. Sirtuins in mammals: insights into their biological function. Biochemical J. 404, 1-13 (2007).

65. Chen, $H$. et al. Role of SIRT1 and AMPK in mesenchymal stem cells differentiation. Ageing Res. Rev. 13, 55-64 (2014).
66. Hwang, J. W., Yao, H., Caito, S., Sundar, I. K. \& Rahman, I. Redox regulation of SIRT1 in inflammation and cellular senescence. Free Radic. Biol. Med. 61 95-110 (2013).

67. Stünkel, W. et al. Function of the SIRT1 protein deacetylase in cancer. Biotechnol. J. 2, 1360-1368 (2007).

68. Takemura, A. et al. Sirtuin 1 retards hyperphosphatemia-induced calcification of vascular smooth muscle cells. Arterioscler Thromb. Vasc. Biol. 31, 2054-2062 (2011).

69. H, Vaziri, S. K. et al. hSIR2(SIRT1) functions as an NAD-dependent p53 deacetylase. Cell 107, 149-159 (2001).

70. Ahmed, M. et al. Humanized Affinity-matured monoclonal antibody $8 \mathrm{H} 9$ has potent antitumor activity and binds to FG loop of tumor antigen B7-H3. J. Biol. Chem. 290, 30018-30029 (2015).

71. Benzon, B. et al. Correlation of B7-H3 with androgen receptor, immune pathways and poor outcome in prostate cancer: an expression-based analysis. Prostate Cancer Prostatic Dis. 20, 28-35 (2017). 